\title{
Differential effects of DMKG on the energy metabolism, autophagy, and apoptosis of the parental and DOX-resistant liver cancer cell lines
}

\author{
Li Wu${ }^{1}$, Tao $\mathrm{Yu}^{1}$, Zihui $\mathrm{Ni}^{1}$, Weiwei Zhang ${ }^{2}$, Yulan Wang ${ }^{2}$, Weidong $\mathrm{Li}^{1}$, Xiao $\mathrm{Liu}^{1}$, and \\ Zhipeng Chen ${ }^{1}$ \\ ${ }^{1}$ Nanjing University of Chinese Medicine School of Pharmacy \\ ${ }^{2}$ Nanjing University of Chinese Medicine
}

April 28, 2020

\begin{abstract}
BACKGROUND AND PURPOSE In this study, we compared the different effect of Dimethyl-2-ketoglutarate (DMKG) on energy metabolism, autophagy, and apoptosis between parental and doxorubicin-resistant liver cancer cell lines. EXPERIMENTAL APPROACH In the energy metabolism section, cell viability, adenosine triphosphate (ATP) level, doxorubicin (DOX) content, energy metabolism phenotype, and enzymes expression were measured. In the autophagy part, autophagy vesicles were observed, enzymes expression and cell viability were detected. Apoptosis rate and expression of enzymes were detected in the apoptosis part. KEY RESULTS DMKG can directly act on ATP synthase and prolyl hydroxylase, affecting the cellular oxidative phosphorylation and glycolysis levels, respectively, in both SMMC-7721 and SMMC-7721/DOX cells. However, its practical effects depend on the function of the mitochondrial malate-aspartate shuttle. Furthermore, ATP-reduced cell autophagy had partial anti-apoptotic effects in SMMC-7721 cells, which is an important reason for the weak inhibition of cell viability observed at low doses of DMKG. In contrast, DMKG showed no significant effect on autophagy but a high cell death rate in SMMC-7721/DOX cells. Induced high expression levels of p-AMPK, p-mTOR, and Bcl-2 could inhibit DMKG-induced apoptosis in SMMC-7721 cells. However, activation of Caspase-3 was the most important molecular effect that was observed in relation to the DMKG-induced apoptosis in SMMC-7721/DOX cells. CONCLUSIONS AND IMPLICATIONS These results suggest that inhibition of energy metabolism can reduce the effects of autophagy in DOX resistant cells, which could improve the therapeutic outcomes and reverse the resistance to chemotherapeutic drugs. This article provides a new idea and method for reversing tumor resistance.
\end{abstract}

\begin{abstract}
Abbreviations
$\alpha$-KG, $\alpha$-Ketoglutaric acid; AOA, Aminooxyacetate; PHD, prolyl hydroxylase; HIF, hypoxia inducible factor; DMKG, Dimethyl-2-ketoglutarate; Sod, Sodium oxamate; L-Mal, L-(-)-Malic acid; 2-ME, 2-Methoxyestradiol; DOX, doxorubicin; Rot, Rotenone; AA, antimycin A; Oli, Oligomycin; Glu, Glucose; TCA cycle, tricarboxylic acid cycle; OXPHOS, oxidative phosphorylation; ATP, adenosine triphosphate; MAS, malateaspartate shuttle; Met, methotrexate; MTT, 3-(4,5-dimethylthiazol-2-yl)-2,5-diphenyltetrazolium bromide; PBS, phosphate buffer; PVDF, polyvinylidene difluoride; PI, propidium iodide; DMEM, Dulbecco's modified Eagle's medium; OCR, Oxygen consumption rate; ECAR, Extra cellular acidification rate; AST, aspartate aminotransferase; LD, lactic acid; HK, hexokinase; LDH, lactate dehydrogenase; mTOR, mammalian target of rapamycin; RAP, rapamycin; AMPK, adenylate activated protein kinase; C-Casp3, Cleaved Caspase-3
\end{abstract}

\section{Introduction}


The reprogramming of energy metabolism widely exists in tumor cells (Vander et al. , 2017). Glycolysis is often abnormally activated, with or without decreased levels of oxidative phosphorylation (OXPHOS), which provides a certain number of ATP molecules to maintain the normal cellular physiology and sufficient intermediate metabolites for biosynthesis (Bayliak et al., 2017). Through a complex feedback regulation, some intermediate metabolites can interfere with OXPHOS or glycolysis to maintain a relative balance between the cellular energy metabolism and material metabolism (Hossain et al., 2019)

SMMC-7721 is a human hepatocarcinoma cell (HCC) line that exhibits characteristic metabolic phenotype of enhanced glycolytic activity, with relative deficiency of mitochondrial energy metabolism and spare capacity of OXPHOS. However, when these cells acquired drug resistance by sustained low dose doxorubicin (DOX) treatment (SMMC-7721/ DOX), the mitochondrial energy metabolism was found to be increased significantly (Abboud et al., 2018). These phenotypic changes in the cellular energy metabolism reflects the activation of tricarboxylic acid cycle (TCA cycle), OXPHOS, malate-aspartate shuttle (MAS), and dynamic changes in a series of intermediates (Haase et al.,2017).

$\alpha$-ketoglutarate $(\mathrm{a}-\mathrm{KG})$ is one of the most important intermediate metabolites of TCA and MAS, and it acts as a feedback regulator of OXPHOS and glycolysis, altering the cellular energy metabolism (Fonsecaet al., 2019). On one hand, $\alpha$-KG can inhibit the activity of ATP synthase in the mitochondria, causing a decrease in the mitochondrial energy metabolism (Tarhonskaya et al., 2014; Fu et al., 2015). On the other hand, $\alpha-K G$ can exert a suppressive effect on glycolysis by enhancing the activity of prolyl hydroxylase (PHD) in the cytoplasm, which is a rate-limiting enzyme in the degradation of hypoxia inducible factor- $1 \alpha$ (HIF-1 $\alpha$ ) (Abbresciaet al., 2012; Wang et al., 2018; Shang et al.,2017). Since the above-mentioned two types of biochemical reactions occur in two different cellular compartments, the mitochondria and cytoplasm, different metabolism and transport rates of a-KG may result in disparate consequences in relation to energy metabolism in different metabolic phenotype cells, and might lead to significantly different outcomes, such as survival or death, autophagy or apoptosis (Yang et al., 2015; Wu et al., 2018). Currently, a-KG is thought to be involved in aging, tumorigenesis, and tumor drug resistance.

Dimethyl 2- ketoglutarate (DMKG) is an a-KG analogue with cell permeability (Wadood et al., 2014; Liuet al., 2010). It is stable and can be metabolized into a-KG after entering the cells. The important question is how does this exogenous a-KG affect the energy metabolism of the SMMC-7721 HCCs with different energy metabolic phenotypes. In this study, SMMC-7721 and SMMC-7721/DOX cells were used to investigate the differential effects of DMKG on energy metabolism, autophagy, and apoptosis to further understand the distinct patterns of energy metabolism between the drug-sensitive and drug-resistant HCCs; our data has also provided new ideas for studying the reversal agents for drug-resistant HCCs.

\section{Methods}

\section{Cell culture}

The SMMC-7721 cell line was purchased from the Cell Bank of the Chinese Academy of Sciences (Shanghai, China), and was grown under standard cell culture conditions in a medium supplemented with $100 \mathrm{U} \cdot \mathrm{ml}^{-1}$ of penicillin, $100 \mathrm{mg} \cdot \mathrm{ml}^{-1}$ of streptomycin, and $10 \%$ foetal bovine serum at $37^{\circ} \mathrm{C}$ in a humidified atmosphere with $5 \% \mathrm{CO}_{2}$.

Drug-resistant SMMC-7721 cells were continuously induced by low-dose $\left(0.25 \mu \mathrm{g} \cdot \mathrm{l}^{-1}\right)$ DOX, and the cells were referred as SMMC-7721/DOX.

Cytotoxicity assays

SMMC-7721 and SMMC-7721/DOX cells $\left(5 \times 10^{4}\right.$ cells $\left.\cdot \mathrm{ml}^{-1}\right)$ were seeded into 96 well plates, allowed to attach overnight, and then treated with DMKG $(0.05-10 \mathrm{mM})$ for $24 \mathrm{~h}$. Cell viability was measured by the MTT assay, and the inhibition rate $(\mathrm{IR})\left(\mathrm{IR}=\left(1-\left(\mathrm{OD}_{\mathrm{drug}}-\mathrm{OD}\right.\right.\right.$ blank $) /\left(\mathrm{OD}_{\text {control }}-\mathrm{OD}\right.$ black $\left.\left.)\right) \times 100 \%\right)$ and the half maximal inhibitory concentration $\left(\mathrm{IC}_{50}\right)$ were calculated using GraphPad Prism. In the rescue experiments, the cells were treated with a combination of DMKG $(5 \mathrm{mM})$ and DOX at varying concentrations 
$(0.1,0.5,8,20,40 \mu \mathrm{M})$ for $24 \mathrm{~h}$. The experiment was repeated 3 times; the mean was obtained from all 3 experiments, and the reversal index (RI) was calculated as follows:

$\mathrm{RI}=\mathrm{IC}_{50}(\mathrm{SMMC}-7721 / \mathrm{DOX}) / \mathrm{IC}_{50}(\mathrm{SMMC}-7721)$.

Intracellular ATP measurements

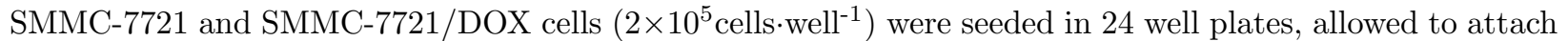
overnight, and then treated with different concentrations of DMKG (0.05-10 mM) for $24 \mathrm{~h}$. Cells were then washed twice with ice-cold PBS and lysed with PBS containing 1\% Triton X-100 at 37 for 1 min. ATP level in the cell lysates was measured using an ATP assay kit and were normalized by protein content.

Distribution of DOX measured by confocal microscopy

SMMC-7721 and SMMC-7721/DOX cells $\left(0.5-1.0 \times 10^{6}\right.$ cells $^{*}$ well $\left.{ }^{-1}\right)$ were cultured in 6 well plates with different drugs for $24 \mathrm{~h}$. DOX has an anthracycline structure, showing red fluorescence. The cells were examined by confocal microscopy at $488 \mathrm{~nm}$ to observe the distribution of DOX.

Mitochondrial stress test and glycolysis stress test

Oxygen consumption rate (OCR, $\left.\mathrm{pmol}^{*} \mathrm{mg}^{-1} \mathrm{pro}\right)$ and extracellular acidification rate (ECAR, $\left.\mathrm{mpH}^{*} \mathrm{mg}^{-1} \mathrm{pro}\right)$ were assessed using the Seahorse XF96 Extracellular Flux Analyser with XF cell mito stress and glycolysis stress test kit (Seahorse Bioscience, North Billerica, MA, USA).

Briefly, SMMC-7721 and SMMC-7721/DOX cells $\left(5 \times 10^{3}\right.$ cells*well $\left.^{-1}\right)$ were seeded in a 96 well cell culture XF microplate, allowed to adhere overnight, and then treated with DMKG $(5 \mathrm{mM})$ alone or with Sod (1 $\mu \mathrm{M})$ or Mal $(1 \mu \mathrm{M})$ for $12 \mathrm{~h}$. The cells were washed with assay medium before incubation with $0.5 \mathrm{ml}$ assay medium for $1 \mathrm{~h}$ at 37 in a $\mathrm{CO}_{2}$-free incubator. Oligomycin (Oli, ATP synthase blocker, $0.5 \mu \mathrm{M}$ ) was used to measure the ATP turnover and to determine the proton leak. The mitochondrial uncouplerFCCP (carbonyl cyanide 4-[trifluoromethoxy] phenylhydrazone, $0.5 \mu \mathrm{M}$ ) was used to measure the maximum respiratory function (maximal OCR). The reserve capacity was calculated as the difference between the maximal OCR and the basal respiration. Rotenone (an inhibitor of complex I, $0.5 \mu \mathrm{M}$ ) and antimycin A (a blocker of complex III, $0.5 \mu \mathrm{M}$ ) were used to measure the non-mitochondrial respiration.

In the glycolytic stress test, the basic glycolytic function of the cells was evaluated using glucose $(10 \mathrm{mM})$ and detecting ECAR. Oli $(1 \mu \mathrm{M})$ was used to inhibit the mitochondrial energy metabolism, and the ECAR was measured to assess the glycolytic capacity and glycolytic reserve. 2-DG (50 $\mathrm{mM}$ ) was used to inhibit glycolysis and assess non-glycolytic acidification. The results were normalized to total cell protein as determined by the BCA assay.

Western blot analyses

SMMC-7721 and SMMC-7721/DOX cells $\left(0.5-1.0 \times 10^{6}\right.$ cells $\cdot$ well $\left.{ }^{-1}\right)$ were cultured in 6 well plates with different drugs for $24 \mathrm{~h}$, and then were collected and washed. The cell extracts were prepared using lysis buffer consisting of RIPA buffer, PMSF(Phenylmethanesulfonyl fluoride), and protease inhibitor (100:1:1). The protein concentration was determined by BCA assay kit (Shanghai Biyuntian Biological Co. Ltd., Shanghai, China). Then the proteins were resolved by SDS-PAGE and transferred onto the polyvinylidene difluoride (PVDF) membrane (Millipore, Massachusetts USA). The proteins of interest were identified using specific primary antibodies and then bound to specific secondary antibodies. The expression levels of the proteins of interest were quantified from their corresponding bands using Image Lab (NIH, Bethesda, MD). The changes in the density of the protein bands were expressed as fold changes in comparison to that of the controls in the blot, after normalization to $\beta$-actin.

Immunofluorescence Assay

SMMC-7721 and SMMC-7721/DOX cells $\left(0.5-1.0 \times 10^{6}\right.$ cells·well $\left.{ }^{-1}\right)$ were cultured in 24 well plates with different drugs for $24 \mathrm{~h}$, and then were collected and washed. The cells were then fixed with $4 \%$ paraformaldehyde for $20 \mathrm{~min}$, blocked, and then washed with PBS. The cells were permeabilized with $0.2 \%$ Triton X-100 in 
PBS at $25^{\circ} \mathrm{C}$ for $10 \mathrm{~min}$ and washed. Then, the cells were incubated with the primary antibody overnight at $37^{\circ} \mathrm{C}$, followed by adding $1 \mathrm{ml}$ of $3 \%$ BSA solution and blocking for 30 min. One microlitre of the antibody against either HIF-1 $\alpha$ (diluted with 1\% BSA in PBS, 1:100) or LC3 (diluted with 1\% BSA in PBS, 1:100) was used for incubation for $2 \mathrm{~h}$. After the cells were washed three times with PBS, secondary antibody was diluted at 1:200 with $1 \% \mathrm{BSA}$ in PBS and applied to the cells for 5 min at $25^{\circ} \mathrm{C}$. Then, washed 4 times with PBS. In the dark, DAPI staining solution was added for nuclear staining, at $37^{\circ} \mathrm{C}$ for $5 \mathrm{~min}$, followed by 3 PBS washes. Appropriate amount of fluorescent anti-quenching agent was put on the glass slide, and the cover glass was placed on the glass slide for detection, and the cells were analysed by fluorescence microscopy.

Flow cytometric analysis

SMMC-7721 and SMMC-7721/DOX cells $\left(0.5-1.0 \times 10^{6}\right.$ cells.well $\left.{ }^{-1}\right)$ were cultured in 6 well plates with different drugs for $24 \mathrm{~h}$, and then were collected, washed, and re-suspended in $500 \mu \mathrm{l}$ of $1 \times$ binding buffer. Subsequently, $5 \mu \mathrm{l}$ of Annexin V-FITC conjugate and $5 \mu \mathrm{l}$ of propidium iodide (PI) were added to the cells, which were then gently vortexed and incubated for $15 \mathrm{~min}$ at 25 in the dark. Thereafter, apoptosis was analysed by flow cytometer (BD Biosciences, USA) within $1 \mathrm{~h}$.

Electron microscopy assay

At the end of the experiment, cells were fixed with a mixture of $2 \%$ formaldehyde and $0.2 \%$ glutaraldehyde in $0.1 \mathrm{M}$ cacodylate buffer $(\mathrm{pH} 7.2)$ for $30 \mathrm{~min}$ at $37^{\circ} \mathrm{C}$. To stop the fixation, free aldehyde groups were blocked by soaking the cells in $50 \mathrm{mM}$ of $\mathrm{NH} 4 \mathrm{Cl}$ in cacodylate buffer for $1 \mathrm{~h}$. After rinsing with the buffer, the cells were mechanically removed, sedimented by centrifugation, enclosed in liquified $2 \%$ agarose, and post-fixed with $1 \%$ osmium tetroxide in distilled water for $1 \mathrm{~h}$, followed by enbloc staining for $1 \mathrm{~h}$ with $1 \%$ aqueous uranyl acetate. After the cell preparations were dehydrated in a series of graded ethanol, embedding it in Epon-araldite. To unambiguously identify the autophagic structures, 10 serial ultrathin sections (80 nm) were prepared on copper slot grids and stained with uranyl acetate and lead citrate and were observed at $80 \mathrm{kV}$ using a Hitachi H-7650 electron microscope. Electron micrographs were acquired with a CCD digital camera.

Data and statistical analysis

The data are presented as mean \pm SD. All statistical analyses were performed using SPSS 15.0 statistical software. Error bars indicate standard error of mean. The differences were considered significant when $\mathrm{p}$ values were less than 0.05 . The analysis of experiments with more than two groups was performed using one-way ANOVA with Scheffe's correction for multiple comparisons.

Materials

DMKG, methotrexate (Met), sodium oxalate, L-(-)-malic acid (L-Mal), and 3-(4,5-dimethylthiazol-2-yl)-2,5diphenyltetrazolium bromide (MTT) were purchased from Sigma Aldrich Chemical Co. (St Louis, MO, USA). DMEM and phosphate buffer (PBS) were purchased from HyClone Corporation. (Logan, Utah, USA). Trypsin (Trypsin-EDTA, 0.25\%), foetal bovine serum (FBS), and antibiotic-antimycotic solution were procured from Gibco Life Technologies Corporation (Grand Island, NY, USA). ATP assay kit and BCA protein assay kit were purchased from Shanghai Biyuntian Biological Co. Ltd. (Shanghai, China). Annexin V-FITC apoptosis detection kit was obtained from Nanjing KeyGen Biotech Co. (Nanjing, China). Mitochondrial stress glycolysis stress test kits were purchased from Seahorse Bioscience (North Billerica, MA, USA).

Nomenclature of targets and ligands

Key protein targets and ligands in this article are hyperlinked to corresponding entries in http://www. guidetopharmacology.org, the common portal for data from the IUPHAR/BPS Guide to PHARMACOLOGY (Harding et al ., 2018), and are permanently archived in the Concise Guide to PHARMACOLOGY 2017/18 (Alexander et al. , 2017a, b).

\section{Results}


$D M K G$ enhances the sensitivity of SMMC-7721 and SMMC-7721/DOX cells to chemotherapy by intervening energy metabolism

In the MTT experiments, a contrasting trend was observed in the cell viability of SMMC-7721 and SMMC7721/DOX cells in response to varying doses of DMKG. Treatment with 0.05-0.5 mM of DMKG for $24 \mathrm{~h}$ showed a weak proliferative effect in both the SMMC-7721 and SMMC-7721/ DOX tumor cells, with growth rates of $8.98 \%$ and $10.06 \%$ at a dose of $0.5 \mathrm{mM}$, respectively. However, above $0.5 \mathrm{mM}$, its proliferative effects disappeared, and it exhibited an inhibitory effect on both SMMC-7721 and SMMC-7721/DOX cells, with growth inhibition of about $10.08 \%$ and $25.95 \%$, respectively, at a dose of $10 \mathrm{mM}$ (PH 7.0) (Figure 1A).

ATP analysis revealed that the ATP level in SMMC-7721/DOX cells was significantly higher than in SMMC7721 cells, and the treatment with DMKG at a nontoxic dose of $1 \mathrm{mM}$ could significantly decrease the ATP level in SMMC-7721/DOX cells (Figure 1B), with an average inhibition rate of $31.42 \%$. However, there was no significant change in the ATP level in SMMC-7721 cells treated with DMKG. In addition, when both the SMMC-7721 and SMMC-7721/ DOX cells were treated with a higher dose of DMKG (10 mM), we observed an average inhibition of $24.15 \%$ in SMMC-7721 cells and 69.08\% in SMMC-7721/DOX cells with respect to that in the control cells treated with Dulbecco's modified Eagle's medium (DMEM) (Figure 1C).

Furthermore, we studied the effect of treatment of DMKG (low toxic dose, $5 \mathrm{mM}$ ) in the presence of varying concentrations of DOX (0.1-40 $\mu \mathrm{M})$ on the viability of SMMC-7721 and SMMC-7721/DOX cells. DMKG was found to increase the inhibitory effects of DOX on both the SMMC-7721 and SMMC-7721/DOX cells at all concentrations tested (Figure 1D), especially in SMMC-7721/DOX cells, which showed a change in $\mathrm{IC}_{50}$ value from $319.8 \mu \mathrm{M}$ to $4.37 \mu \mathrm{M}$. As is evident through confocal microscopy, DMKG could promote the aggregation of DOX (red fluorescent) in both the SMMC-7721 and SMMC-7721/DOX cells remarkably (Figure 1E).

These results indicated that DMKG could interfere with the energy metabolism of both SMMC-7721 and SMMC-7721/DOX HCCs at a non-toxic dose, and its actual effect was associated with the differences in the basic metabolic status of these cells, which resulted in enhanced sensitivity of SMMC-7721/DOX cells to chemotherapeutic agents. Intervening energy metabolism may be a good strategy to reverse the drug resistance of HCCs, and it is necessary to further study the mechanism by which DMKG can intervene in the energy metabolism in the SMMC-7721/DOX cells. 
A

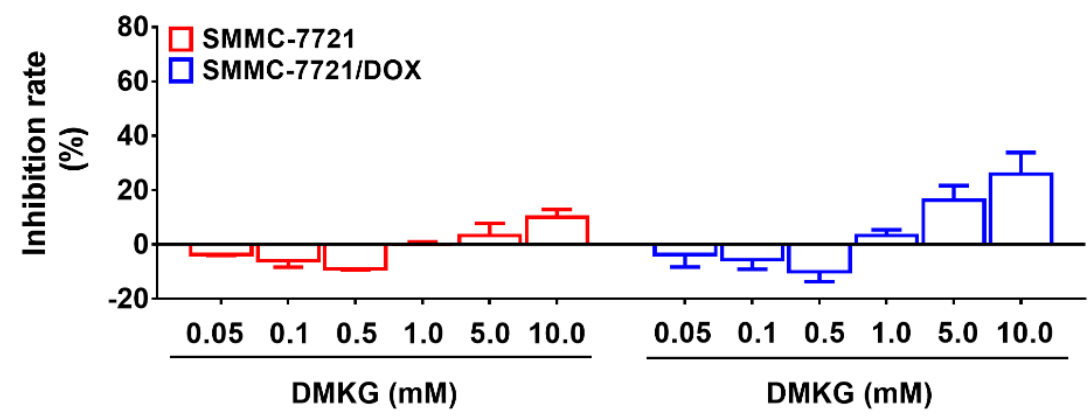

B

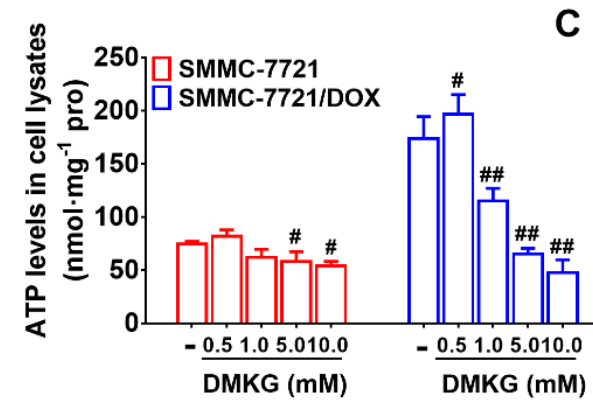

C

D

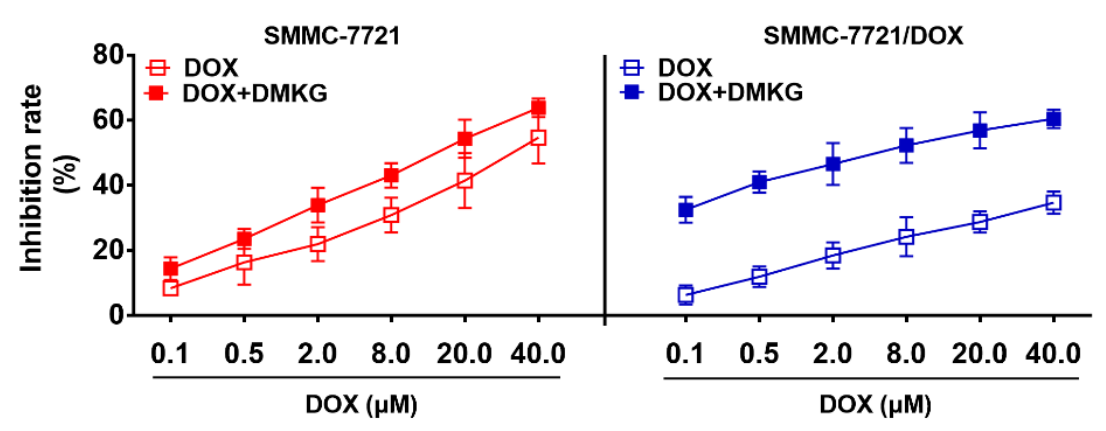

E

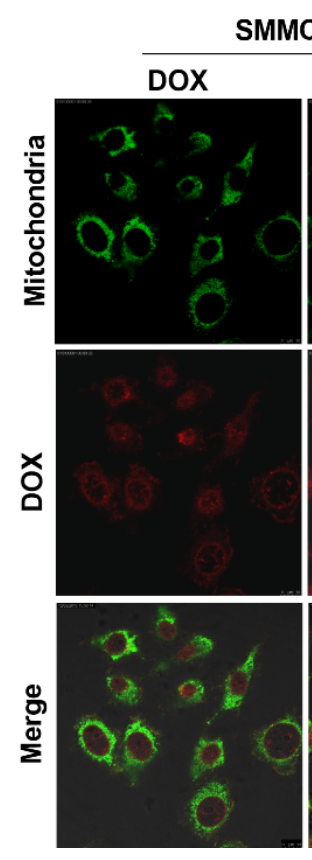

MMC-7721

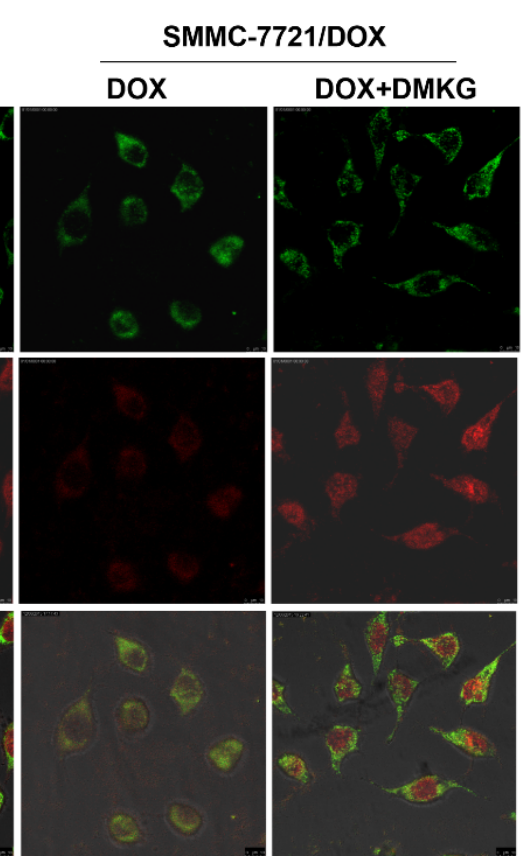




\section{Figure 1}

DMKG enhances the sensitivity of SMMC-7721 and SMMC-7721/DOX cells to DOX by intervening energy metabolism. (A) Changes in cell viability induced by DMKG treatment at doses $0.05-50 \mathrm{mM}$ ( $n=6$ per group). (B) Changes in the ATP level induced by DMKG treatment $(0.5-50 \mathrm{mM})(n=3$ per group). (C) Inhibition of ATP in both SMMC-7721 and SMMC-7721/DOX cells $(n=3$ per group). (D) Changes in cell viability induced by treatment with varying doses of DOX $(0.1-40 \mu \mathrm{M})$ with DMKG $(5 \mathrm{mM})(n=6$ per group). (E) Distribution of DOX (red fluorescence) in the cells as assessed by confocal microscopy. ${ }^{\#} P$ $<0.05, \# \# P<0.01$, significantly different from control group.

$D M K G$ caused a stronger inhibition in the mitochondrial energy metabolism in SMMC-7721/DOX cells than $S M M C-7721$ cells

In order to study the mechanism of differential changes in the ATP level of SMMC-7721 and SMMC7721/DOX cells caused by DMKG treatment, the energy metabolism phenotype tests were performed; the results showed that DMKG can lead to a significant inhibition in the glycolysis and OXPHOS in both the SMMC-7721 and SMMC-7721/DOX cells. However, the quantitative analysis of metabolic phenotype revealed that the trend in the changes were different between the SMMC-7721 and SMMC-7721/DOX cells (Figure 2).

In mito stress test, SMMC-7721/DOX cells showed a higher level of basal respiration, ATP production, maximal respiration, and spare capacity than that in the SMMC-7721 cells. Treatment with ATP synthetase inhibitor oligomycin (Oli) could decrease the levels of oxygen consumption rate, particularly in SMMC-7721/DOX cells (Figure 2A, B). It is interesting that DMKG almost eliminated the effect of Oli on mitochondrial respiration in both the SMMC-7721 and SMMC-7721/DOX cells (Figure 2A, solid circles). These results suggested that DMKG might act on the same target as Oli, the ATP synthetase. Quantitative analysis showed DMKG caused a greater inhibition on the ATP production capacity in SMMC-7721/DOX cells as compared to that in the SMMC-7721 cells (Figure 2B).

In glycolytic stress test, although the basal glycolysis analysis suggested similar levels in both the SMMC7721 and SMMC-7721/DOX cells, the maximum glycolysis of SMMC-7721/DOX cells was significantly higher than that of the SMMC-7721 cells. In contrast to the results of the mito stress test, DMKG showed a stronger inhibition on the basal glycolysis in SMMC-7721 cells, with a similar effect on the maximum glycolysis as compared to that in the SMMC-7721/DOX cells (Figure 2C, D).

Energy metabolic phenotype analysis suggested that the main energy metabolism mode in SMMC-7721 cells was glycolysis, and DMKG mainly inhibited glycolysis in these cells. SMMC-7721/DOX cells were found to be stronger in the mitochondrial energy metabolism than the SMMC-7721 cells, and DMKG could inhibit both the mitochondrial energy metabolism and glycolysis in the SMMC-7721/DOX cells (Figure 2E). 
A

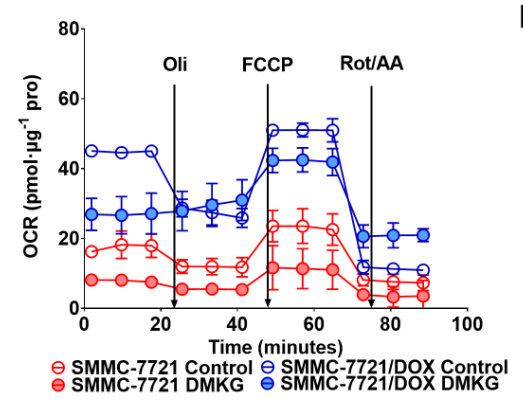

C

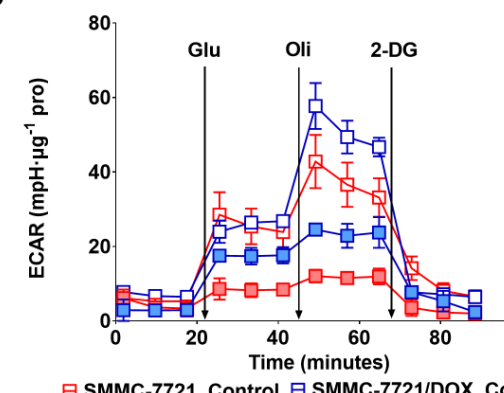

B ${ }_{80}$ प SMMC-7721 Control QSMMC-7721/DOX Control SMMC-7721 DMKG DSMMC-77211DOX DMKG
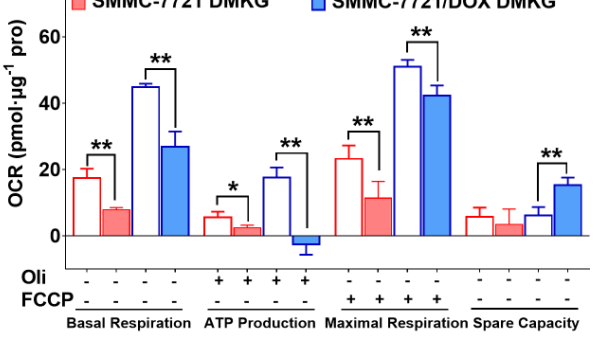

D

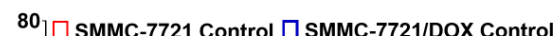

SMMC-7721 Control $\square$ SMMC-7721/DOX Control SMMC-7721 DMKG SMMC-7721/DOX DMKG

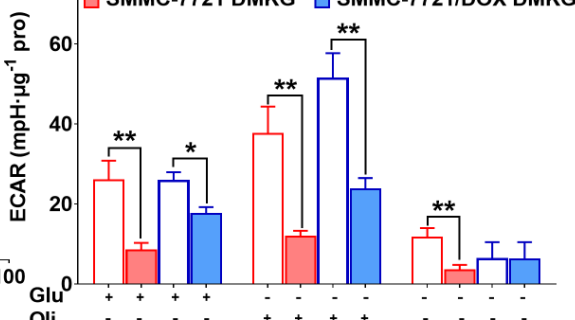

旦 SMMC-7721 Control 星 SMMC-7721/DOX Control
SMMC-7721 DMKG

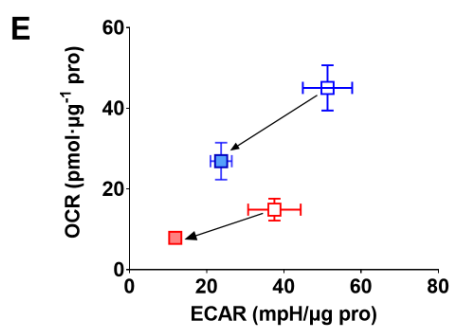

口 SMMC-7721 Control a SMMC-7721/DOX Control

\section{Figure 2}

DMKG caused a stronger inhibition on mitochondrial energy metabolism in SMMC-7721/DOX than SMMC7721 cells. (A) Oxygen consumption rate (OCR) based on the mito stress test ( $n=3$ per group). (B) Quantitative analysis of the basal respiration, ATP production, maximum OCR, and spare capacity $(n=3$ per group). (C) Extra cellular acidification rate (ECAR) of the culture media in the glycolysis stress test $(n$ $=3$ per group). (D) Quantitative analysis of the basal glycolysis, glycolytic capacity and glycolytic reserve ( $n=3$ per group). (E) OCR against ECAR in SMMC-7721 and SMMC-7721DOX. ${ }^{*} P<0.05,{ }^{* *} P<0.01$, significantly different from control groups.

Effect of DMKG on mitochondrial energy metabolism depends on the function of the malate-aspartate shuttle

It is well known that the transport of a-KG between the cytoplasm and mitochondria depends on the malateaspartate shuttle (MAS). To examine if MAS accounts for the differential effects of DMKG on the energy metabolic phenotype of the SMMC-7721 and SMMC-7721/DOX cells, the energy metabolism phenotypes were determined using DMKG along with MAS inhibitor aminooxyacetate (AOA).

Firstly, AOA $(0.8 \mathrm{mM})$ was used to block the transfer of $\alpha-\mathrm{KG}$ between the mitochondria and cytoplasm by inhibiting the activity of aspartate aminotransferase (AST) (Figure 3A), and then the changes in the energy metabolism induced by DMKG were studied. The results show that AOA could effectively reverse the inhibition of ATP induced by DMKG in SMMC-7721/DOX cells (Figure 3B), but had no obvious effects in the SMMC-7721 cells. 
In mito stress test, AOA predominantly reversed the inhibition of DMKG on basal respiration and maximal respiration in the SMMC-7721/DOX cells (Figure 3C, D, grey filled blue circles and histograms). However, it had little effect on the OCR of SMMC-7721 cells (Figure 3C, D, grey filled red circles and histograms).

In glycolysis stress test, surprisingly, there was no significant change in glycolysis in both the SMMC7721 cells and SMMC-7721/DOX cells (Figure 3E, F, grey filled circles and histograms). According to the metabolic phenotype analysis, AOA could reverse the effect of DMKG on mitochondrial energy metabolism in SMMC-7721/DOX cells but did not have significant effect on SMMC-7721 cells (Figure. 3G).

In agreement with our previous studies (Wu et al., 2018), the expression of malic dehydrogenase (MDH, the key enzyme of MAS) in SMMC-7721/DOX was significantly higher than that in SMMC-7721 cells (Figure $3 \mathrm{H}$ ), which provided a better understanding of the effect of AOA in rescuing the inhibition of DMKG on the mitochondrial energy metabolism in SMMC-7721/DOX cells while exhibiting little effect in SMMC-7721 cells.

A

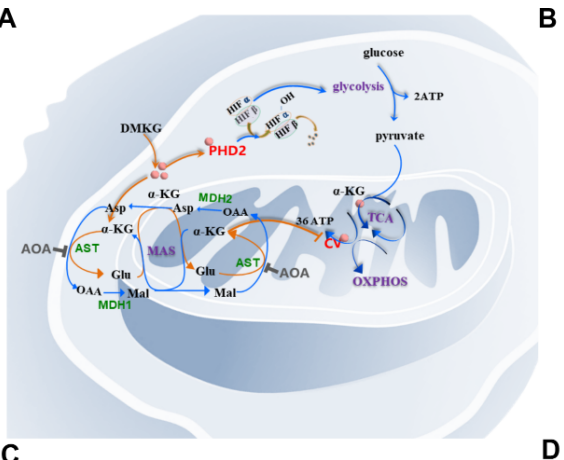

C

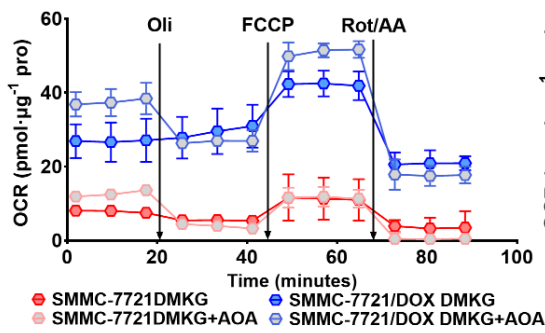

E

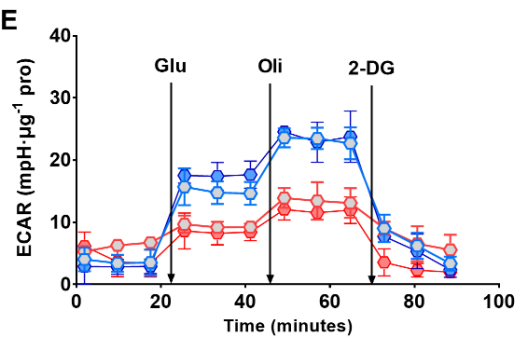

$\ominus$ SMMC-7721 DMKG $\theta$ SMMC-7721/DOX DMKG $\because$ SMMC-7721 DMKG+AOA $\ominus$ SMMC-7721/DOX DMKG+AOA

G

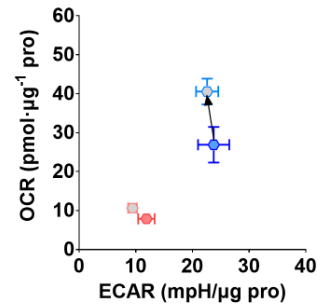

$\mathbf{F}$

H

SMMC-7721 DMKG SMMC-7721/DOX DMKG
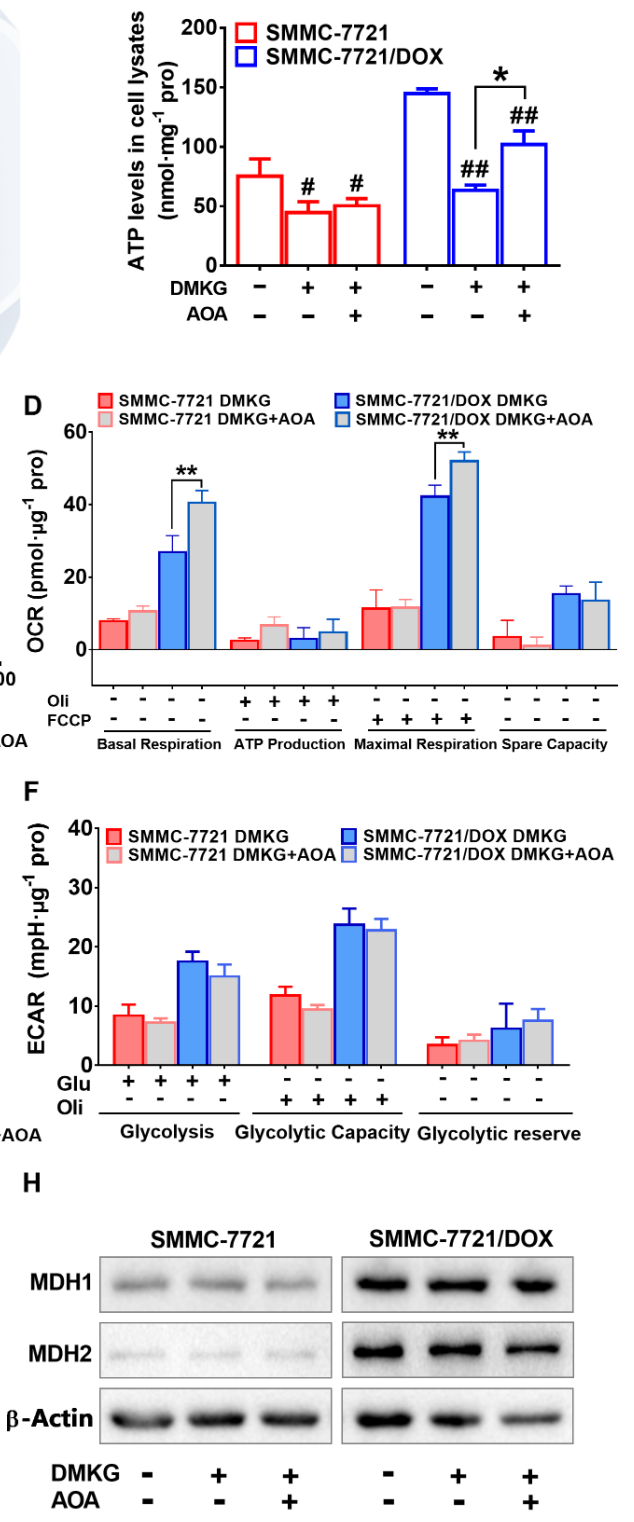


\section{Figure 3}

Different states of MAS involved in the DMKG-induced energy metabolism changes. (A) Schematic ideograms of variations in the distribution of a-KG induced by AOA. (B) Changes in ATP level induced by DMKG and AOA ( $n=3$ per group). (C) Effects of AOA and DMKG on OCR of both the cells in mito stress test $(n=3$ per group). (D) Quantitative analysis of the basal respiration, ATP production, maximum OCR, and spare capacity ( $n=3$ per group). (E) Effects of AOA and DMKG on ECAR of the culture media in the glycolysis stress test ( $n=3$ per group). (F) Quantitative analysis of the basal glycolysis, glycolytic capacity and glycolytic reserve ( $n=3$ per group). (G) Changes of OCR against ECAR in both the cells induced by DMKG and AOA. $(\mathrm{H})$ Protein expression of MDH1 and MDH2 in both the SMMC-7721 and SMMC-7721/DOX cells. ${ }^{*} P<0.05,{ }^{\#} P<0.01$, significantly different from control group; ${ }^{*} P<0.05$, ${ }^{* *} P<0.01$, significantly different from DMKG group.

\section{Effect of DMKG on glycolysis depends on PHD activity in the cytoplasm}

The results described above demonstrated that blocking the mitochondrial transfer of a-KG could partly reverse the inhibition of OXPHOS induced by DMKG in SMMC-7721/DOX cells. However, the inhibition of glycolysis was not aggravated, inconsistent with the predicted effect of AOA that further activated PHD, and thereby decreased the level of glycolysis. Thus, we further examined the effects of DMKG and AOA on the metabolites and enzymes involved in the glycolysis of both the cells, including lactic acid (LD) levels, PHD enzyme activity, and HIF-1 $\alpha$, hexokinase (HK), and lactate dehydrogenase (LDH) levels.

First, LD levels in the intracellular and cell culture supernatants indicated that DMKG could significantly reduce the production of $\mathrm{LD}$ in both the cells (Figure 4A), and the inhibitory effects were more pronounced in the SMMC-7721 cells (75.2\% inhibition) than in the SMMC-7721/DOX cells (45.1\% inhibition). However, the release rates of LD were similar in all groups, and these effects were not further suppressed by AOA treatment (Figure 4B).

The results of PHD showed that DMKG had different effects on the enzyme activities of the two cells, especially in the cell culture supernatants. Specifically, PHD activity in the control groups of both the cells was similar, and DMKG could enhance the PHD activity intracellularly in both the cells (Figure $4 \mathrm{C})$. Although the rate of increase in total PHD activity in SMMC-7721/DOX (99.9\%) was higher than that in the SMMC-7721 cells $(33.6 \%)$, most of the enzymes in SMMC-7721/DOX cells were released outside of the cell (Figure 4D), and the rate of increase in the intracellular enzyme activity was lower than that of the SMMC-7721 cells. Thus, AOA treatment had no significant effect on the DMKG induced changes in the intracellular PHD activity. These results suggested that DMKG may increase the cell membrane permeability through other means, leading to PHD leakage and weakening its inhibitory effect on glycolysis.

The level of HIF-1 $\alpha$ in both the cells was detected by immunofluorescence and western blotting. The results showed that DMKG decreased the intensity of HIF-1 $\alpha$ staining as compared to that in the control cells (Figure 4E), and its expression level was significantly decreased (Figure 4F, G). Furthermore, we observed that SMMC-7721/DOX cells showed morphological changes, with a thinner and longer phenotype in the DMKG treated group, suggesting that the cell state was poor. No severe changes were observed in the AOA group of both the cells.

In line with the results described above, the expression of the downstream targets of HIF-1 $\alpha$ (Figure $4 \mathrm{~J}$ ), HK and LDH demonstrated decreased expression upon DMKG treatment (Figure 4F-I). 
A

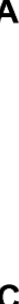

C
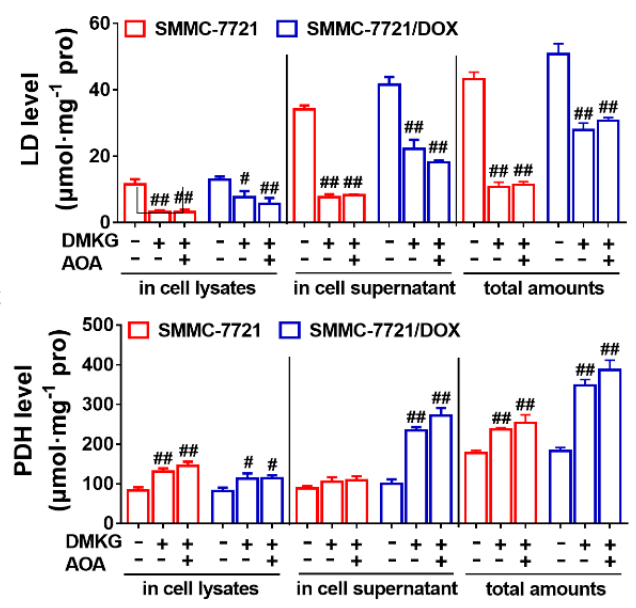

E

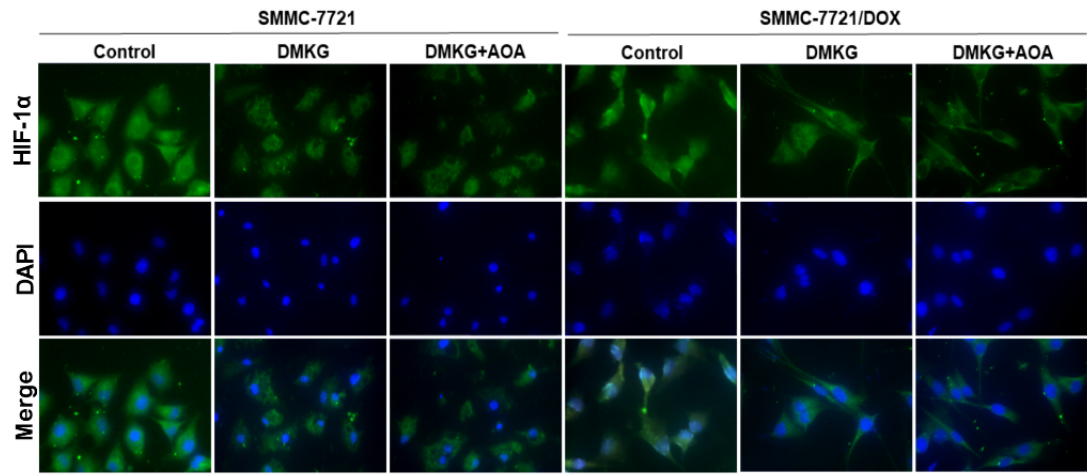

$\mathbf{F}$

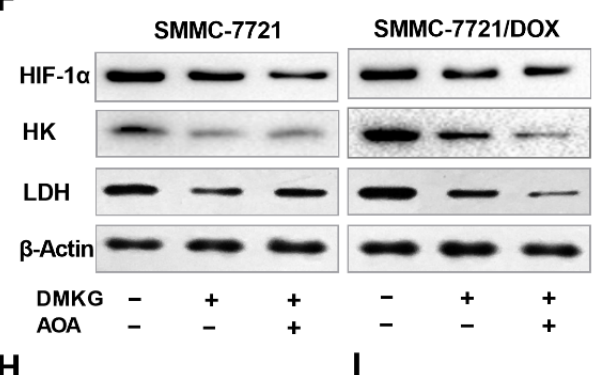

H

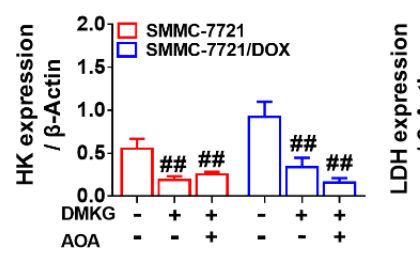

B
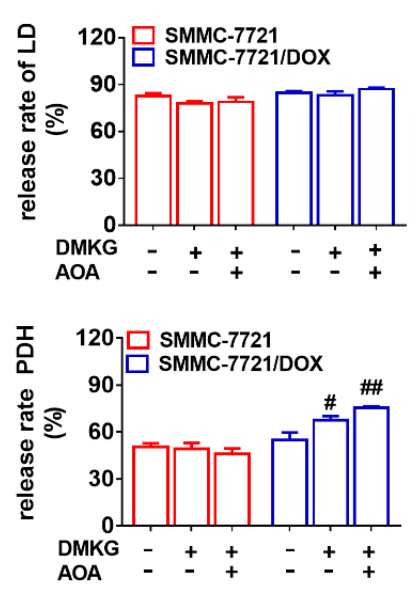

G

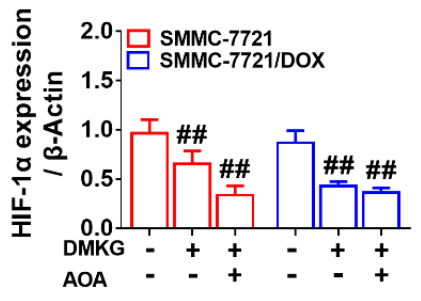

$\mathbf{J}$

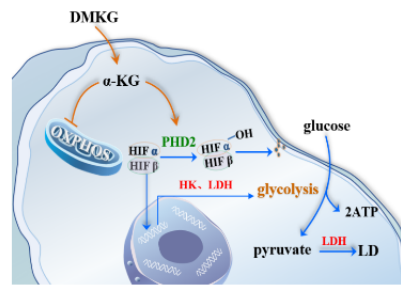

Figure 4

Effect of DMKG on glycolysis depends on the PHD activity in the cytoplasm. (A) LD levels in cell lysates and cell supernatant induced by DMKG and AOA ( $n=3$ per group). (B) Release rate of LD ( $n=3$ per group). (C) PHD levels in cell lysates and cell supernatant induced by DMKG and AOA ( $n=3$ per group). (D) Release rate of PHD ( $n=3$ per group). (E) HIF- $1 \alpha$ level detected by immunofluorescence. (F) Protein expression of HIF-1 $\alpha$, HK, and LDH in both the SMMC-7721 and SMMC-7721/DOX cells ( $n=3$ per group). (G-I) Quantitative analysis of HIF-1 $\alpha$, HK, and LDH in both the cells ( $n=3$ per group). (J) Schematic 
ideograms of the action of DMKG on glycolysis. ${ }^{\#} P<0.05$, ${ }^{\#} P<0.01$, significantly different from control group.

Effect of DMKG treatment on autophagy of the SMMC-7721 and SMMC-7721/DOX cells

Autophagy is an important regulatory mechanism for maintaining the homeostasis under cellular energy stress. When energy is plentiful and the ATP/ AMP ratio is high, the mammalian target of rapamycin (mTOR) is activated and inhibits autophagy. In contrast, reduced intracellular ATP levels could inactivate mTOR, which in turn promotes autophagy. Therefore, we studied the effect of DMKG on the autophagy of the two cells.

The results showed that unlike the SMMC-7721 cells, the autophagic vacuoles occur in the control SMMC7721/DOX cells (Figure 5A), with positive expression of autophagic proteins LC3 (punctate green fluorescence, Figure 5B). Quantitative analysis of LC3 I and II also confirmed that the autophagy was activated in the SMMC-7721/DOX cells (Figure 5C-E). It is well known that the cells can gain energy through autophagy, which may be another important reason for the high level of energy metabolism in SMMC-7721/DOX cells.

It is because of these differences that a large distinction in the effects of DMKG on autophagy of the two cells was observed. Specifically, DMKG promoted the onset of autophagy in SMMC-7721 cells, with the appearance of vacuolar bilayer membrane structure (Figure 5A), enhanced spot green brightness of LC3 (Figure 5B), high expression of LC3 I and II, and high level of LC3 II/I ratio (Figure 5C-F).

However, the promotion of autophagy by DMKG did not occur in the SMMC-7721/DOX cells. Autophagy also existed in the SMMC-7721/DOX cells after incubation with DMKG, but the cell damage was observed, and the cell rupture and autophagosome appeared in the intercellular fluid (Figure 5A, red arrow mark). The results of western blotting showed a downward trend in the LC3 II expression. Furthermore, autophagy inhibitor (3-MA, $2.5 \mu \mathrm{M})$ reversed the DMKG induced autophagy, especially in the SMMC-7721 cells. 
A

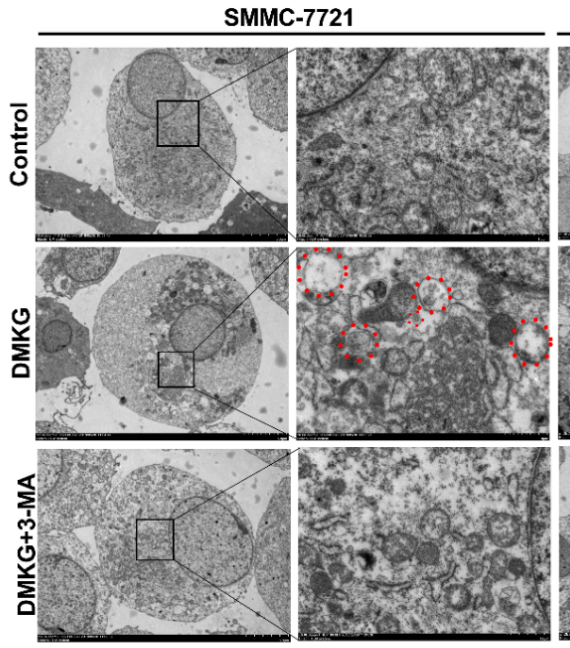

B

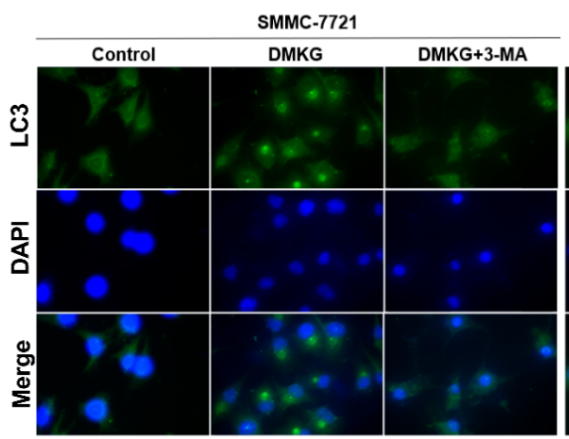

C

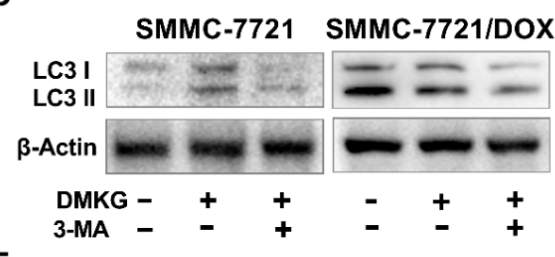

E

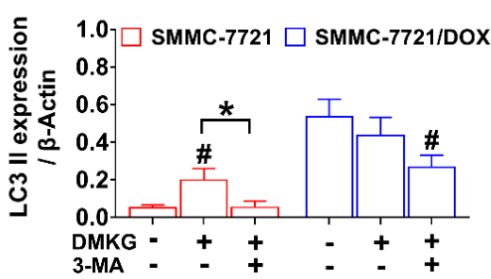

SMMC-7721/DOX

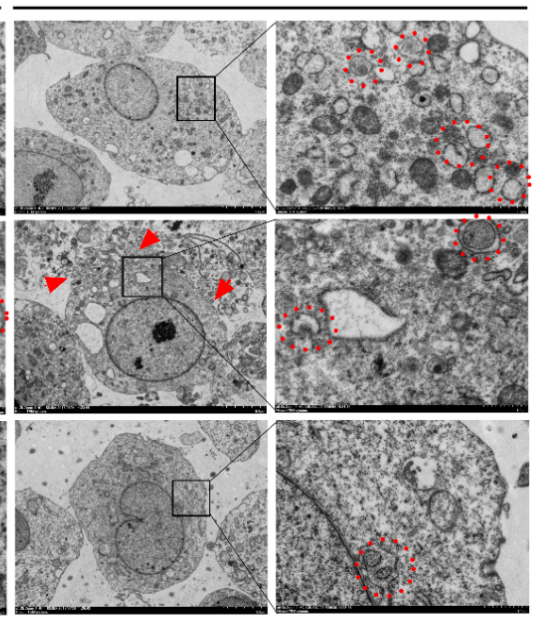

\begin{tabular}{ccc}
\multicolumn{3}{c}{ SMMC-7721/DOX } \\
\hline Control & DMKG & DMKG+3-MA
\end{tabular}

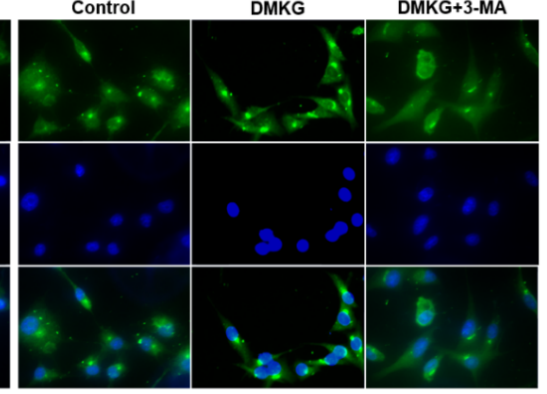

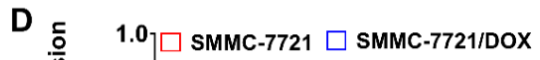

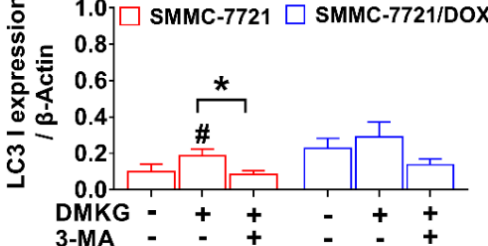

$\mathbf{F}$

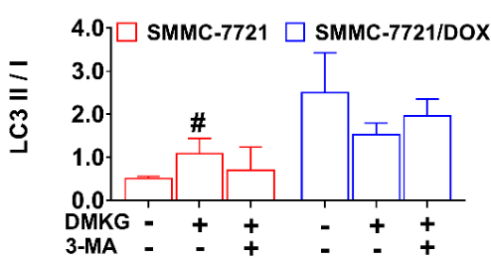

\section{Figure 5}

Effect of DMKG on the autophagy of SMMC-7721 and SMMC-7721/DOX cells. (A) Representative electron micrographs of autophagic vesicles in SMMC-7721 and SMMC-7721/DOX cells. (B) LC3 detection by immunofluorescence. (C) Protein expression of LC3 I and LC3 II in both the cells ( $n=3$ per group). (D-F) Quantitative analysis of LC3 I, LC3 II, and LC3 II/LC3 I ( $n=3$ per group). ${ }^{*} P<0.05$, significantly different from control group; ${ }^{*} P<0.05$, significantly different from DMKG group.

Autophagy affects the pro-apoptotic role of DMKG in the SMMC-7721 and SMMC-7721/DOX cells

A growing number of studies have shown that autophagy plays a dual role in the regulation of cell death. Although autophagy induces apoptosis under certain conditions, some other studies have shown that au- 
tophagy has an inhibitory effect on apoptosis (Ravanan et al., 2017; Jinet al., 2018; Wu et al., 2016). Our data showed that autophagy present in the SMMC-7721/DOX control cells, which differed significantly from that in the SMMC-7721 cells, was beneficial in maintaining high levels of ATP, supporting homeostasis, and inhibiting apoptosis in continuous low dose DOX medium.

Autophagy inhibitor (3-MA, $2.5 \mu \mathrm{M}$ ) and activator rapamycin (RAP, $5 \mathrm{nM}$ ) were used to study the effects of DMKG mediated autophagy on apoptosis in both the cells. The results of viability assay showed that the inhibition of autophagy had no effect on the cell viability in SMMC-7721 cells but could significantly inhibit the cell proliferation in SMMC-7721/DOX cells (inhibition rate 21.84\%); activating autophagy could induce proliferation in the both cells, and the proliferation rate reached $47 \%$ in SMMC-7721 cells, which was higher than that of $15.14 \%$ in the SMMC-7721/DOX cells (Figure 6A).

The results combining DMKG with other drugs suggested that 3-MA enhanced the inhibitory effect of DMKG by inhibiting the autophagy in the SMMC-7721 cells (Figure 6B). However, RAP promoted cell proliferation even in the presence of DMKG. In SMMC-7721/DOX cells, 3-MA had no significant effect on the DMKG mediated inhibition, and RAP was able to rescue the effect on cell viability by DMKG (Figure $6 \mathrm{~B})$. These results suggested that DMKG reduced the ATP levels while triggering autophagy in SMMC-7721 cells, thus, partially offsetting its effects on cell viability. However, in SMMC-7721/DOX cells, DMKGmediated cell death was not significantly associated with autophagy. We speculated that it was related to apoptosis caused by ATP reduction. It was striking that DMKG alone had no effect on apoptosis, but induced apoptosis when administered in combination with 3-MA in the SMMC-7721 cells (Figure 6C, D). In SMMC-7721/DOX cells, DMKG alone induced cell apoptosis (Figure 6C, D) and 3-MA enhanced its proapoptotic effect. The transmission electron microscopy (TEM) images revealed morphological features of apoptosis with typical shrinkage, chromatin condensation (red arrow), and loss of plasma membrane integrity in the SMMC-7721 cells, and more typical nuclear collapse, apoptotic body formation (red arrow), and even cell membrane defects (black arrows) in the SMMC-7721/DOX cells (Figure 6E). 

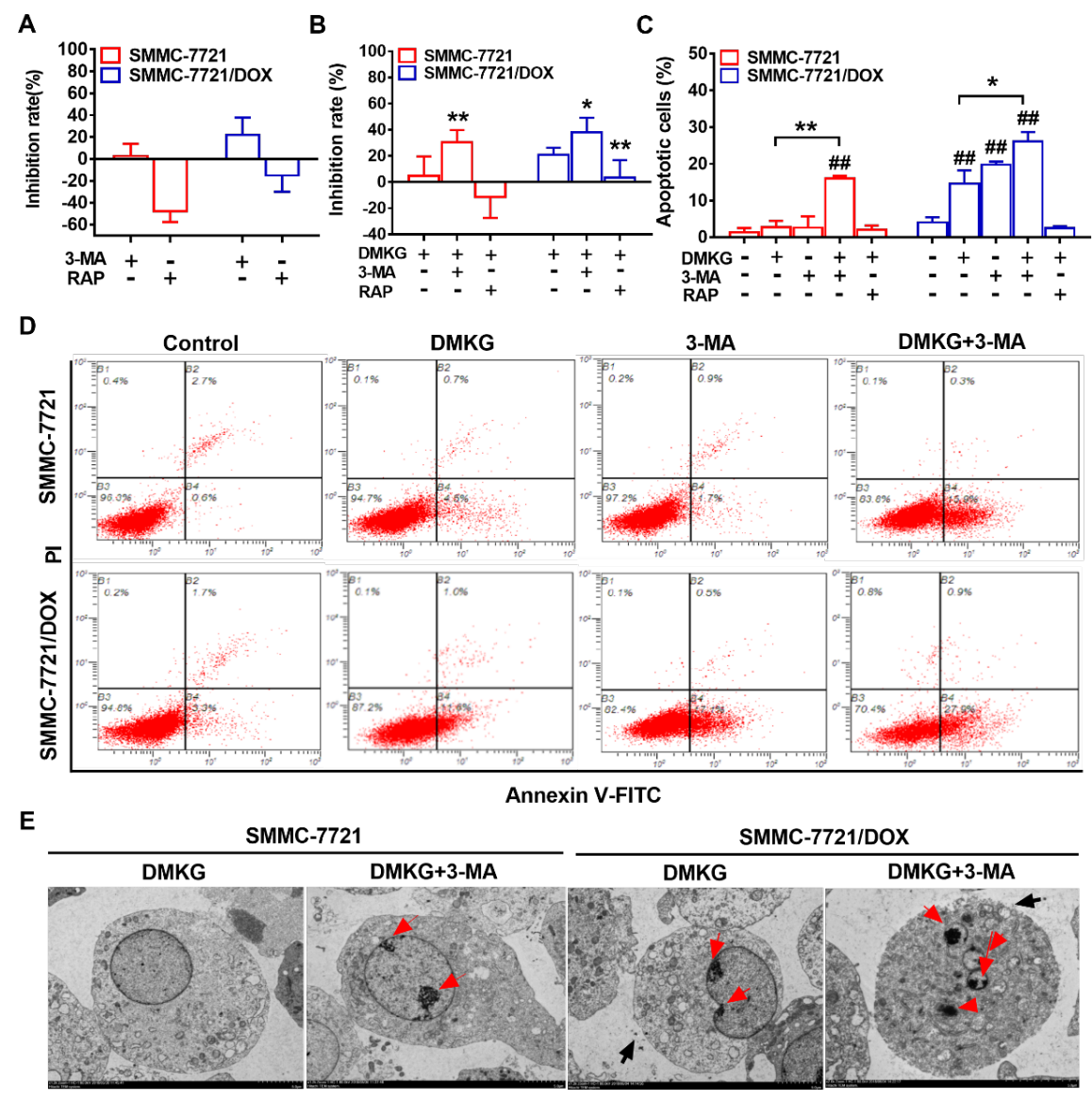

\section{Figure 6}

Autophagy affects the pro-apoptotic role of DMKG in SMMC-7721 and SMMC-7721/DOX cells. (A) Cell viability changes induced by 3 -MA or RAP ( $n=6$ per group). (B) Cell viability changes induced by DMKG with 3-MA or RAP ( $n=6$ per group). (C) Apoptotic rate of SMMC-7721 and SMMC-7721/DOX cells induced by DMKG with 3-MA or RAP ( $n=3$ per group). (D) Flow cytometric analyses of apoptotic cells. (E) Representative electron micrographs of apoptotic cells of SMMC-7721 and SMMC-7721/DOX cells induced by DMKG or with 3-MA. \#\# $P<0.01$, significantly different from control group; ${ }^{*} P<0.05,{ }^{* *} P$ $<0.01$, significantly different from DMKG group.

Inhibition of autophagy aggravates DMKG induced apoptosis by different mechanisms in the SMMC-7721 and SMMC-7721/DOX cells

Under energy stress, adenylate activated protein kinase (AMPK), as the key energy sensor, not only maintains the energy balance by regulating the cell metabolism, but also can regulates autophagy and decay apoptosis, which determines the survival and death of energy stress cells. Specifically, on one hand, it can inhibit the activation of mTOR, relieve its inhibition on Beclin-1, and promote cell autophagy (Figure 7A, panel 1 and 2); on the other hand, it can relieve the inhibition of apoptosis by Bcl-2 (Figure 7A, panel 3) and induce apoptosis. However, whether the cells choose autophagy or apoptosis depends mainly on the activation of the autophagy protein Beclin-1 or apoptotic effector Bcl-2, proapoptotic protein Bax, and the Caspase family proteins.

To explore different mechanisms involved in the effect of DMKG on autophagy/ apoptosis in the SMMC7721 and SMMC-7721/DOX cells, protein levels of p-AMPK, p-mTOR, and Bcl-2 in both the cells were 
analysed. Simultaneously, p-AMPK, Bcl-2 inhibitors CC, ABT, and 3-MA were used in combination with DMKG to observe their effects on the expression of Beclin-1 and Cleaved Caspase-3 (C-Casp3).The results suggested that in SMMC-7721 control cells, the expression of p-AMPK was low with high expression levels of p-mTOR, Bcl-2, and low levels of downstream molecule Beclin-1. Conversely, in SMMC-7721/DOX control cells, p-AMPK was significantly activated, and the protein expression levels of mTOR, p-mTOR, and Bcl-2 were low, but with high expression levels of Beclin-1, which could explain in part the different levels of autophagy in SMMC-7721 and SMMC-7721/DOX control cells (Figure 7A, B).

In addition, DMKG had different effects on the expression of autophagy/ apoptosis related proteins in the two cells. Although similar effects of p-AMPK were induced by DMKG in both the cells (Figure 7C, D), contrasting effects of the expression levels of Beclin-1 and autophagy due to different basal levels of mTOR (Figure 7B, D) were observed. Specifically, in the SMMC-7721 cells, the expression of Beclin-1 was increased, which promoted the onset of autophagy (Figure 7B, F). However, in SMMC-7721/DOX cells, Beclin-1 levels did not change significantly. When p-AMPK was inhibited by CC, p-mTOR, Beclin-1 expression levels showed a reverse trend, while Bcl-2 inhibitor ABT had a weaker effect on autophagy in the SMMC-7721 cells. In SMMC-7721/DOX cells, Beclin-1 was significantly reduced by DMKG with CC or ABT (inhibiting p-AMPK or Bcl-2), although the expression of p-mTOR was low. It is suggested that in SMMC-7721 cells, DMKG regulated autophagy through various pathways such as p-AMPK/ mTOR, p-AMPK/ Beclin-1, pAMPK/ Bcl-2 (Figure 7A panel 1-3). In SMMC-7721/DOX cells, DMKG regulates cell autophagy mainly through p-AMPK/ Beclin-1 (Figure 7A panel 2).

Caspase-3 is a key executor of the apoptotic program. It is cleaved and activated during apoptosis and is necessary for the formation of apoptotic bodies. In this study, DMKG did not have any impact on the levels of C-Casp3 in SMMC-7721 cells, however, by coadministration with p-AMPK, Bcl-2 inhibitor or directly inhibiting autophagy, DMKG could increase the expression of C-Casp3 significantly, and 3-MA, which directly inhibits autophagy, had the strongest effect (Figure 7B, G). In SMMC-7721/DOX cells, DMKG alone could significantly increase the expression of C-Casp3, and CC or ABT could not modify the effects of DMKG. However, 3-MA enhanced the DMKG-induced C-Casp3 expression significantly (Figure 7B, G). These results suggest that p-AMPK, p-mTOR, and Bcl-2 play a certain role in inhibiting DMKG-induced apoptosis in SMMC-7721 cells. However, the expression of these molecules is relatively lower in SMMC7721/DOX cells and activated Caspase-3 is the most important molecular effect related to DMKG-induced apoptosis in SMMC-7721/DOX cells. 
A
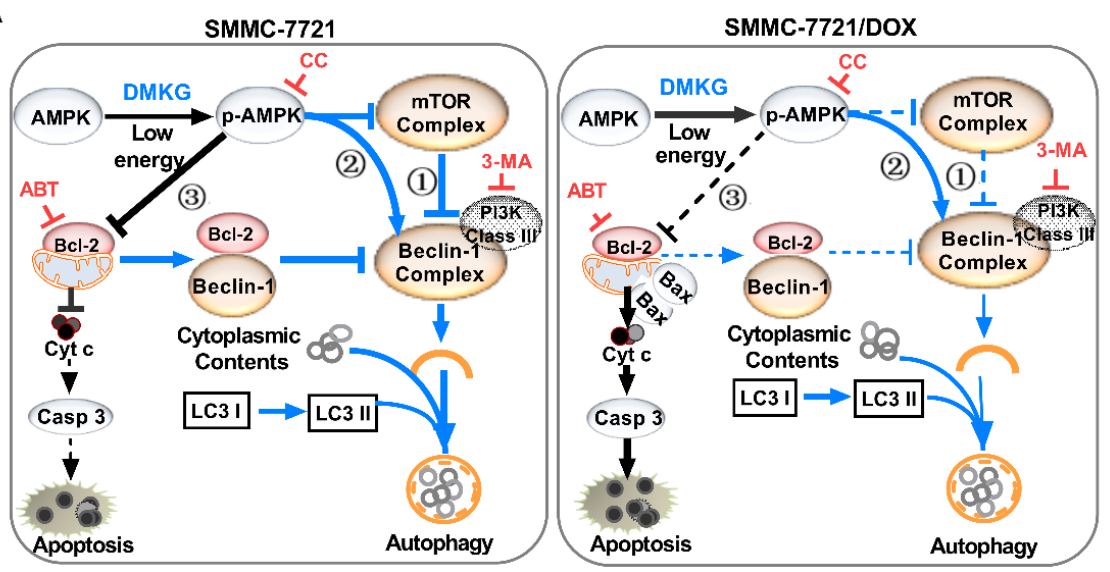

B

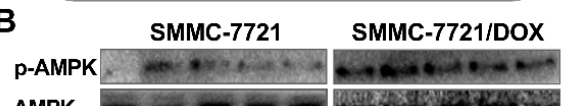

C

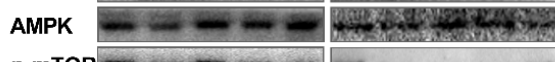

p-mTOR - - - - - -

mTOR $=-\cdots-\cdots-\cdots$

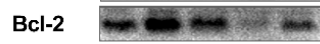

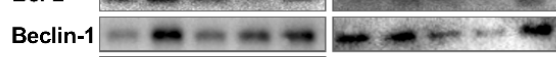

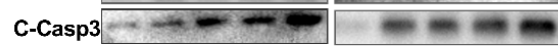

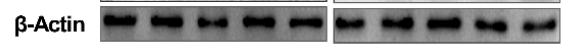

DMKG - + + + + $+++++\mathrm{E}$

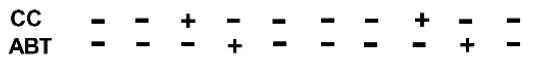

$\begin{array}{llllllllllll}\text { ABT } & - & - & - & + & - & - & - & - & + & - \\ \text { 3-MA } & - & - & - & - & + & - & - & - & - & +\end{array}$

D
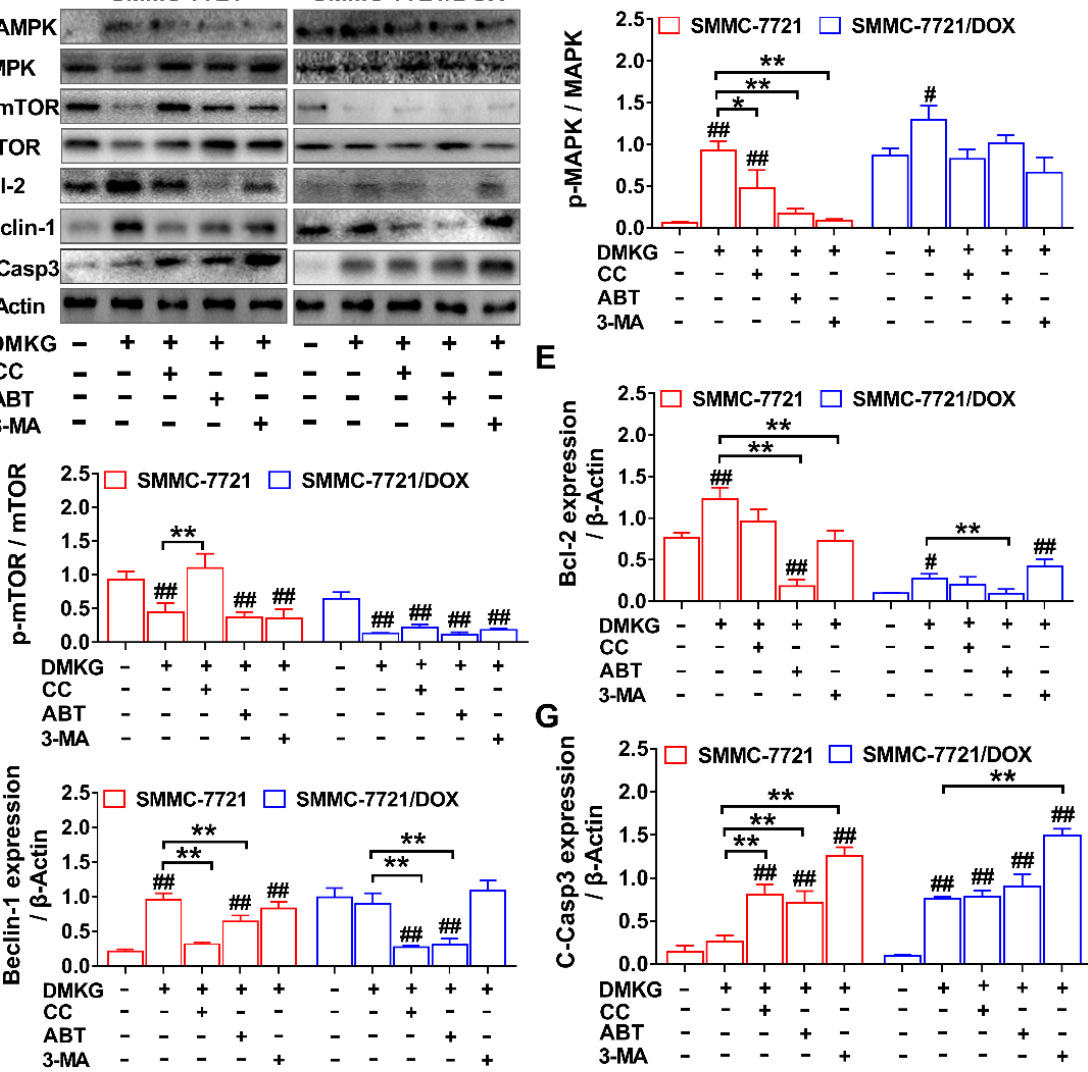

\section{Figure 7}

Inhibition of autophagy aggravates DMKG-induced apoptosis by different mechanisms in SMMC-7721 and SMMC-7721/DOX cells. (A) Schematic ideograms of the effect of DMKG on autophagy and apoptosis in SMMC-7721 and SMMC-7721/DOX cells. (B) Protein expression of p-AMPK, AMPK, p-mTOR, mTOR, Bcl-2, Beclin-1, and C-Casp3 in both the cells ( $n=3$ per group). (C-G) Quantitative analysis of p-AMPK, pmTOR, Bcl-2, Beclin-1, and C-Casp3 in both the cells ( $n=3$ per group). ${ }^{\#} P<0.05$, $\# \# P<0.01$, significantly different from control group; ${ }^{*} P<0.05,{ }^{* *} P<0.01$, significantly different from DMKG group.

\section{Discussion}


An increasing number of studies have shown that small molecular intermediate metabolites can actively participate in the regulation of cell metabolism, proliferation, differentiation, autophagy, apoptosis, and other cellular processes. In this study, we have shown that in SMMC-7721 cells, the effect of the inhibition of mitochondrial $\alpha-\mathrm{KG}$ on ATP synthase resulted in the uncoupling of OXPHOS from the TCA cycle and failure of the mitochondrial bioenergetics, and glycolysis became the main energy metabolism pathway. However, in DOX-resistant SMMC-7721 cells, a remarkable reduction in the cellular $\alpha$-KG levels because of the active MAS and OXPHOS was shown to collaborate with glycolysis in energy metabolism, with higher level of ATP (Wu et al., 2018). These results suggested that intervening energy metabolism may be a potential therapeutic target for reversing the acquired drug resistance.

Thus, DMKG treatment was done to observe the effects of supraphysiological concentration of $\alpha-\mathrm{KG}$ on the cell metabolism, proliferation, and cell death in SMMC-7721 and SMMC-7721/DOX cells. Notably, the energy metabolism of both the SMMC-7721 and SMMC-7721/DOX cells was inhibited, however, that of the SMMC-7721/DOX was more effectively inhibited by DMKG, which was closely related to its dual inhibition on both the ATP synthase and PHD, and reduction in the ATP production. In addition, the present study confirmed that the degree of inhibition of OXPHOS and glycolysis by DMKG depends on the activity levels of the MAS, which further affects its regulation of cell viability, cell death, and drug resistance.

Although, similar inhibition of energy metabolism in response to DMKG treatment was observed in the SMMC-7721 cells, however, the viability of SMMC-7721 cells was not significantly affected. It is well known that autophagy is an important regulatory mechanism that maintains the homeostasis under cellular energy stress conditions by causing two distinct effects on the vital activities of the cells (Lin, 2019; Buszczak et al., 2019). On one hand, autophagy provides abundant nutrients and energy to the cells by degrading the cytoplasmic lysosomes, which promotes cell survival. On the other hand, excessive autophagy can trigger cell death (Chenet al., 2019; D'Arcy, 2019). In recent years, studies have found that the process of tumor development is often accompanied by abnormal autophagy, which in turn impacts the disease progression to some extent. Specifically, during the early stages of tumorigenesis, autophagy inhibits tumor development by eliminating tumorigenic metabolites, inhibiting chronic inflammation, and regulating oncogene-induced senescence. In the advanced stages of tumor development, autophagy leads to tumor resistance by exerting anti-apoptotic effects (Jezek et al., 2010; Fitzwalter et al., 2018). Therefore, the mechanism of cell death was explored from the perspective of autophagy and apoptosis. A high level of autophagy was found in the control group of SMMC-7721/DOX cells. However, this phenomenon does not appear in SMMC-7721 cells because of different levels of p-AMPK and p-mTOR. Autophagy induced by DMKG in SMMC-7721 cells was beneficial for the cell survival, and this effect was due to high expression of anti-apoptotic regulator protein Bcl-2. Unlike SMMC-7721 cells, DMKG-induced apoptosis in SMMC-7721/DOX cells was not significantly associated with autophagy, and the mechanism was thought to involve increased cytochrome $\mathrm{c}$ release and Caspase-3 activation due to mitochondrial dysfunction.

In conclusion, we found that DMKG could suppress the activities of ATP synthase and PHD, respectively, affect cellular OXPHOS and glycolysis. However, its actual effect is dependent on the function of the mitochondrial MAS. It is worth noting that the energy stress-induced autophagy could be advantageous for survival in the SMMC-7721 cells, which is the main reason for the weak inhibition of cell viability by low doses of DMKG. In contrast, DMKG-induced autophagy could exert a synergistic effect on the pro-apoptotic effect with DOX in DOX resistant cells. These results suggest that the inhibition of the energy metabolism in combination with the administration of chemotherapeutic drugs may be an effective method for reversing the tumor resistance.

\section{Acknowledgments}

This work was supported by the National Natural Science Foundation of China $(81873287,81503308)$, the Key Project of Natural Science Research in Colleges and Universities in Jiangsu Province (18KJA360009), Qin Lan Project for Young Academic Leaders of university in Jiangsu Province.

\section{Author Contributions}


L.W., T.Y. and Z.H.N. designed the research. T.Y., W.W.Z. and Y.L.W. carried out the experiments. T.Y. and Z.H.N. analysed the data. L.W. drafted the article. W.D.L., X.L., and Z.P.C. provided fundings. All authors have read and agreed to the published version of the manuscript.

\section{Conflicts of Interest}

The authors declare no conflicts of interest.

\section{Declaration of transparency and scientific rigour}

This Declaration acknowledges that this paper adheres to the principles for transparent reporting and scientific rigour of preclinical research recommended by funding agencies, publishers and other organisations engaged with supporting research.

\section{References}

Abboud MI, McAllister TE, Leung IKH, Chowdhury R, Jorgensen C, Domene Cet al . (2018). 2-Oxoglutarate regulates binding of hydroxylated hypoxia-inducible factor to prolyl hydroxylase domain 2. Chem Commun (Camb) 54: 3130-3133.

Abbrescia DI, La Piana G, Lofrumento NE (2012). Malate-aspartate shuttle and exogenous NADH/cytochrome c electron transport pathway as two independent cytosolic reducing equivalent transfer systems. Arch Biochem Biophys 518: 157-163.

Alexander SPH, Fabbro D, Kelly E, Marrion NV, Peters JA, Faccenda Eet al . (2017a). The Concise Guide to PHARMACOLOGY 2017/18: Enzymes. Br J Pharmacol 174: S272-S359.

Alexander SPH, Peters JA, Kelly E, Marrion NV, Faccenda E, Harding SDet al . (2017b). The Concise Guide to PHARMACOLOGY 2017/18: Ligand-gated ion channels. Br J Pharmacol 174: S130-S159.

Bayliak MM, Lylyk MP, Shmihel HV, Sorochynska OM, Semchyshyn OI, Storey JM et al. ( 2017). Dietary alpha-ketoglutarate promotes higher protein and lower triacylglyceride levels and induces oxidative stress in larvae and young adults but not in middle-aged Drosophila melanogaster. Comp Biochem Physiol A Mol Integr Physiol 204: 28-39.

Buszczak M, Krämer H (2019). Autophagy Keeps the Balance in Tissue Homeostasis. Dev Cell 49: 499-500

Chen X, Xu S, Zhao C, Liu B (2019). Role of TLR4/NADPH oxidase 4 pathway in promoting cell death through autophagy and ferroptosis during heart failure. Biochem Biophys Res Commun 516: 37-43.

D'Arcy MS (2019). Cell death: a review of the major forms of apoptosis, necrosis and autophagy. Cell Biol Int 43: 582-592.

Fitzwalter BE, Towers CG, Sullivan KD, Andrysik Z, Hoh M, Ludwig Met al . (2018). Autophagy Inhibition Mediates Apoptosis Sensitization in Cancer Therapy by Relieving FOXO3a Turnover. Dev Cell 44:555-565 e3.

Fonseca J, Moradi F, Maddalena LA, Ferreira-Tollstadius B, Selim S, Stuart JA (2019). Resveratrol integrates metabolic and growth effects in PC3 prostate cancer cells-involvement of prolyl hydroxylase and hypoxia inducible factor-1. Oncol Lett 17: 697-705.

Fu X, Chin RM, Vergnes L, Hwang H, Deng G, Xing Y et al . (2015). 2-Hydroxyglutarate Inhibits ATP Synthase and mTOR Signaling. Cell Metab 22: 508-515.

Haase VH (2017). HIF-prolyl hydroxylases as therapeutic targets in erythropoiesis and iron metabolism. Hemodial Int 21(Suppl 1): S110-S124.

Harding SD, Sharman JL, Faccenda E, Southan C, Pawson AJ, Ireland Set al . (2018). The IUPHAR/BPS guide to pharmacology in 2018: updates and expansion to encompass the new guide to immunopharmacology. Nucl Acids Res 46: D1091-D1106. 
Hossain SM, Shetty J, Tha KK, Chowdhury EH (2019). $\alpha$-Ketoglutaric Acid-Modified Carbonate Apatite Enhances Cellular Uptake and Cytotoxicity of a Raf- Kinase Inhibitor in Breast Cancer Cells through Inhibition of MAPK and PI-3 Kinase Pathways. Biomedicines 7: E4.

Jezek P, Plecitá-Hlavatá L, Smolková K, Rossignol R (2010). Distinctions and similarities of cell bioenergetics and the role of mitochondria in hypoxia, cancer, and embryonic development. Int J Biochem Cell Biol 42: 604-622.

Jin S, Wei J, You L, Liu H, Qian W (2018). Autophagy regulation and its dual role in blood cancers: A novel target for therapeutic development (Review). Oncol Rep 39: 2473-2481.

Lin PH (2019). Advances in Autophagy, Tissue Injury, and Homeostasis: Cells Special Issue. Cells 8: E743.

Liu H, Shen Q, Zhang J, Fu W (2010). Evaluation of various inverse docking schemes in multiple targets identification. J Mol Graph Model 29: 326-330.

Ravanan P, Srikumar IF, Talwar P (2017). Autophagy: The spotlight for cellular stress responses. Life Sci 188: 53-67.

Shang W, Wei X, Ying W (2017). Malate-aspartate shuttle inhibitor aminooxyacetic acid blocks lipopolysaccharidesinduced activation of BV2 microglia. Int J Physiol Pathophysiol Pharmacol 9: 58-63.

Tarhonskaya H, Chowdhury R, Leung IK, Loik ND, McCullagh JS, Claridge TDet al . (2014). Investigating the contribution of the active site environment to the slow reaction of hypoxia-inducible factor prolyl hydroxylase domain 2 with oxygen. Biochem J 463: 363-372.

Vander Heiden MG, DeBerardinis RJ (2017). Understanding the Intersections between Metabolism and Cancer Biology. Cell 168: 657-669.

Wadood A, Riaz M, Jamal SB, Shah M (2014). Interactions of ketoamide inhibitors on HCV NS3/4A protease target: molecular docking studies. Mol Biol Rep 41: 337-345.

Wang T, Yao W, Li J, He Q, Shao Y, Huang F (2018). Acetyl-CoA from inflammation-induced fatty acids oxidation promotes hepatic malate-aspartate shuttle activity and glycolysis. Am J Physiol Endocrinol Metab 315: E496-E510.

Wu L, Zhao J, Cao K, Liu X, Cai H, Wang J et al . (2018). Oxidative phosphorylation activation is an important characteristic of DOX resistance in hepatocellular carcinoma cells. Cell Commun Signal 16: 6.

Wu X, Feng X, Zhao X, Ma F, Liu N, Guo H et al . (2016). Role of Beclin-1-Mediated Autophagy in the Survival of Pediatric Leukemia Cells. Cell Physiol Biochem 39: 1827-1836.

Yang H, Zhou L, Shi Q, Zhao Y, Lin H, Zhang M et al . (2015). SIRT3-dependent GOT2 acetylation status affects the malate-aspartate NADH shuttle activity and pancreatic tumor growth. EMBO J 34: 1110-1125. 
A

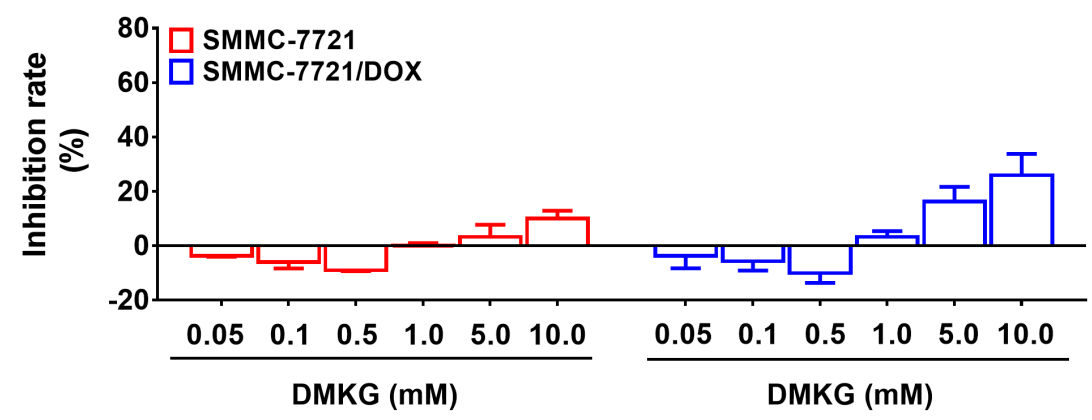

B

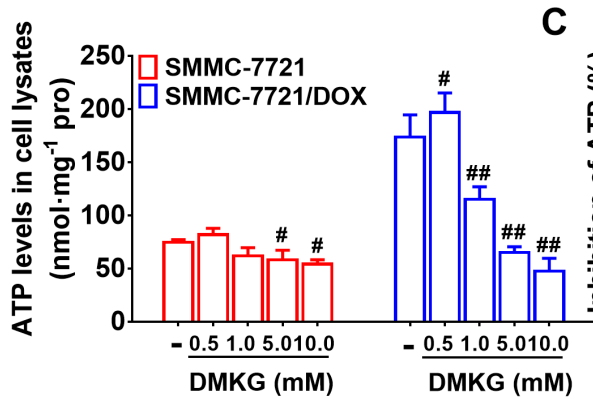

C

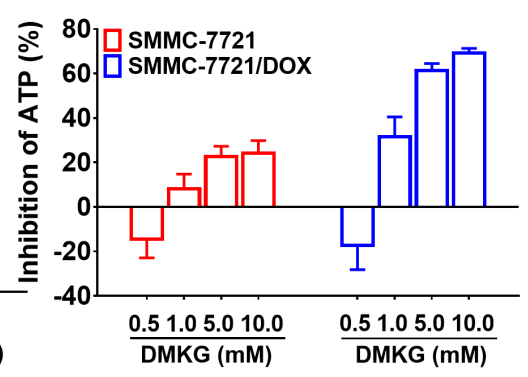

D

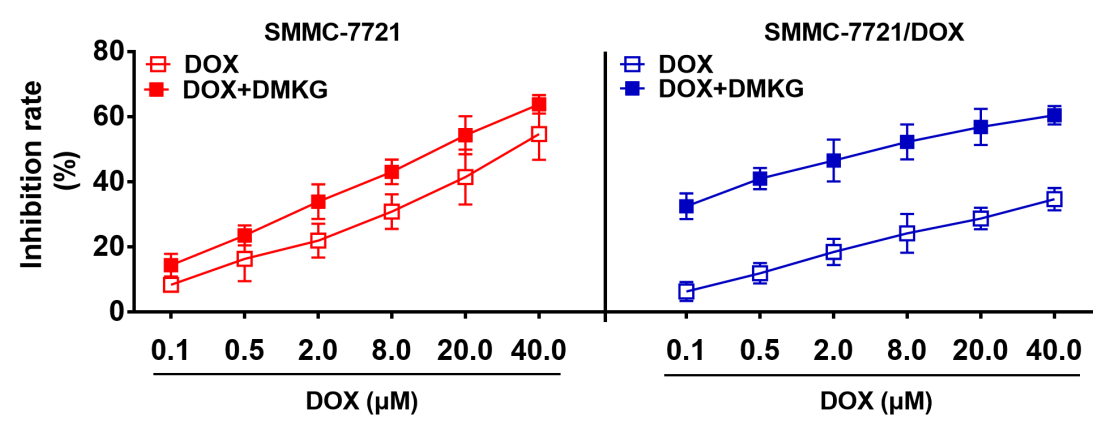

E

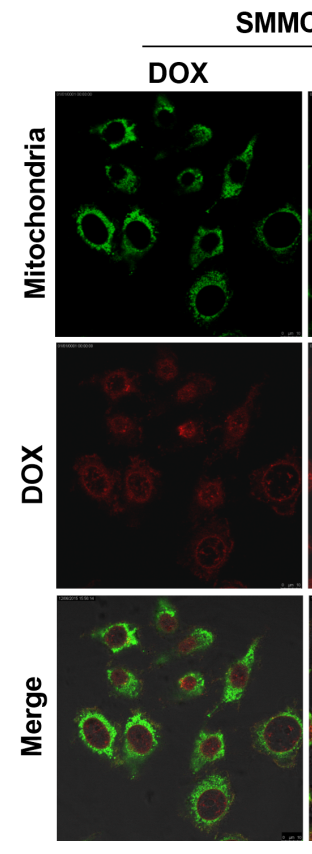

SMMC-7721

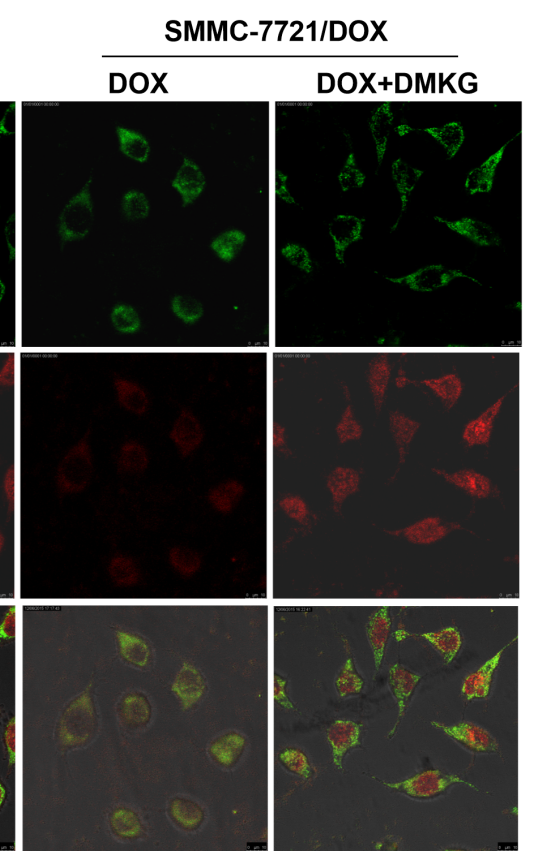


A

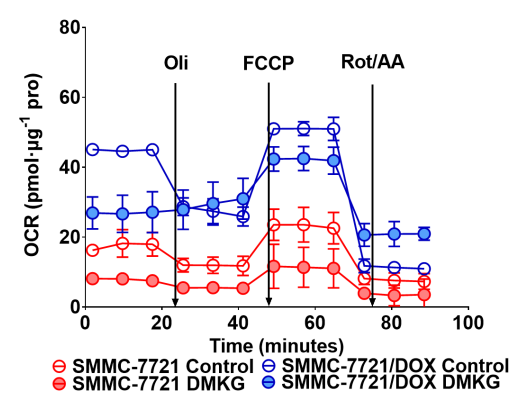

B

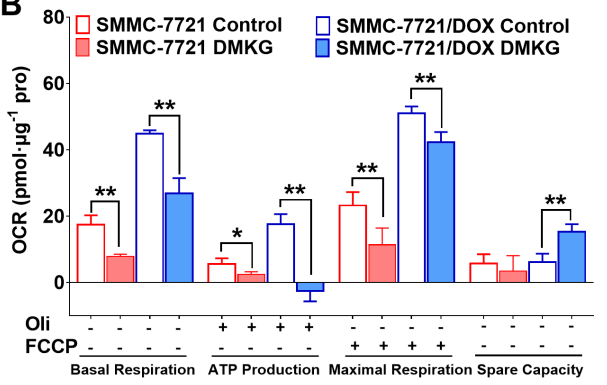

D

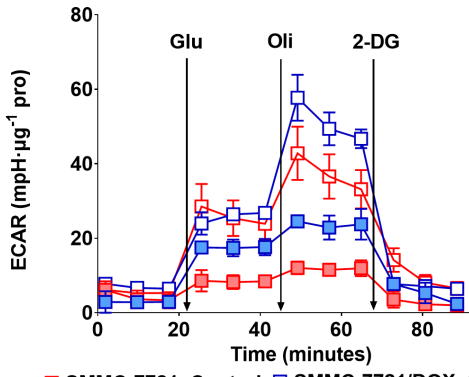

${ }^{80} \square$ SMMC-7721 Control $\mathrm{C}$ SMMC-7721/DOX Control \begin{tabular}{l}
$\square$ SMMC-7721 DMKG $\square$ SMMC-7721/DOX DMKG \\
\hline SMM
\end{tabular}

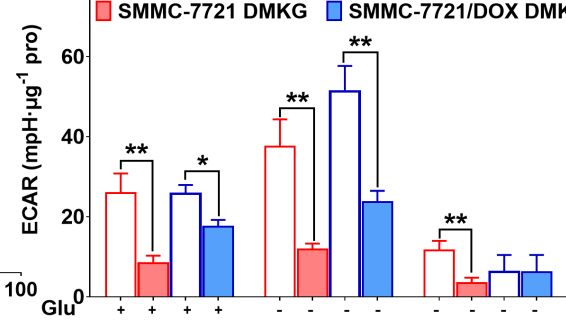
ESMMC-7721 Control $\mathrm{E}$ SMMC-7721/DOX Control GMMC-7721 DMKG SMMC-7721/DOX DMKG

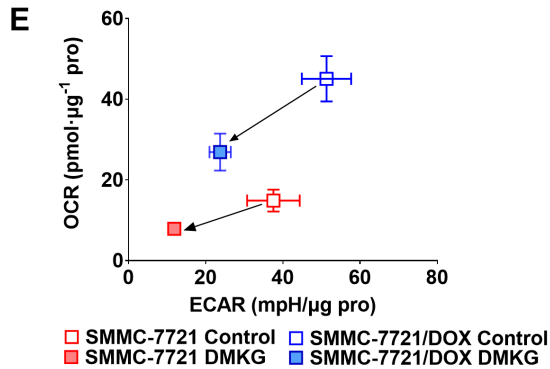


A

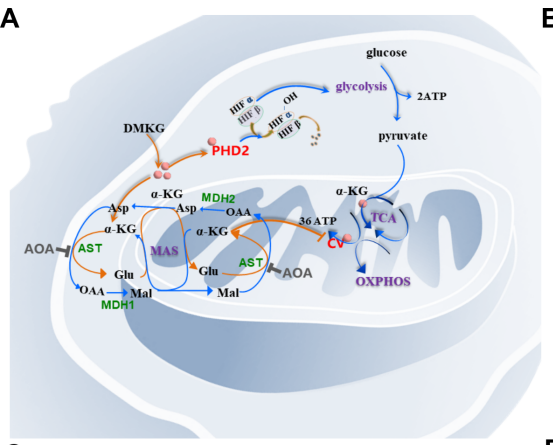

C

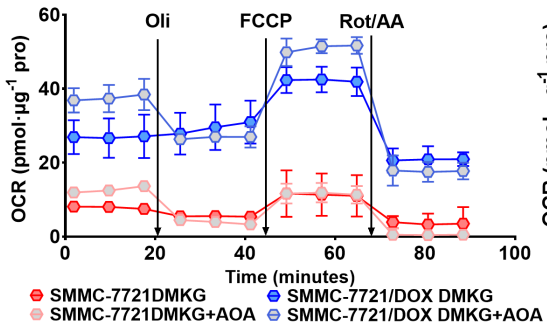

E

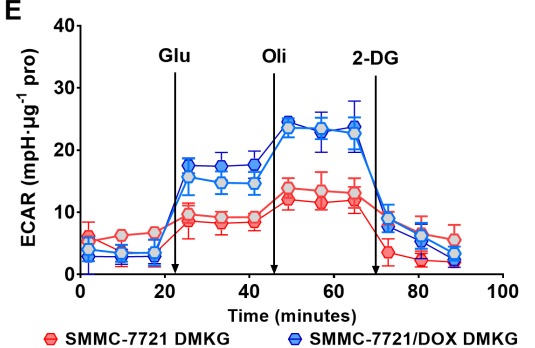

$\ominus$ SMMC-7721 DMKG $\ominus$ SMMC-7721/DOX DMKG

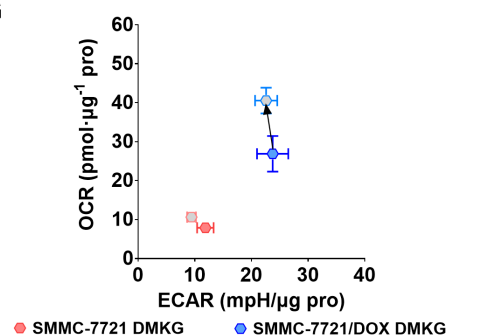

B

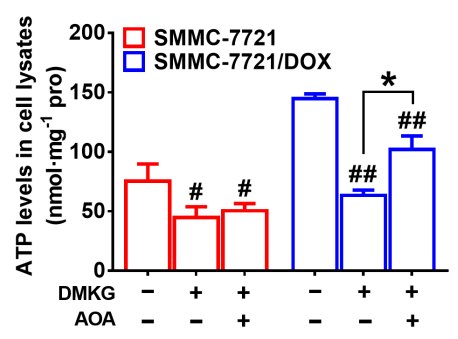

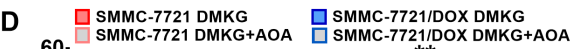

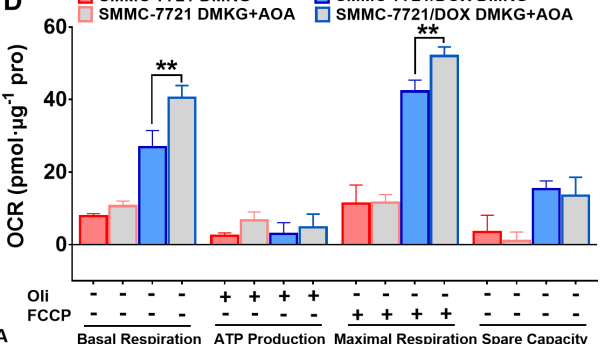

$F$

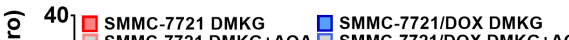

SM 30 SMMC-7721 DMKG+AOA SMMC-7721/DOX DMKG+AOA

ㅁำ

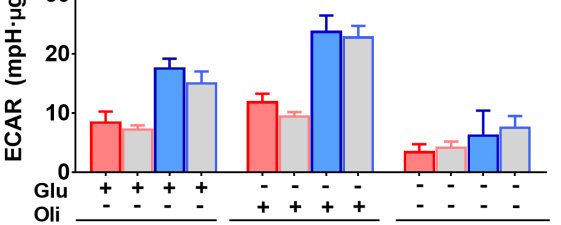

Glycolysis Glycolytic Capacity Glycolytic reserve

H

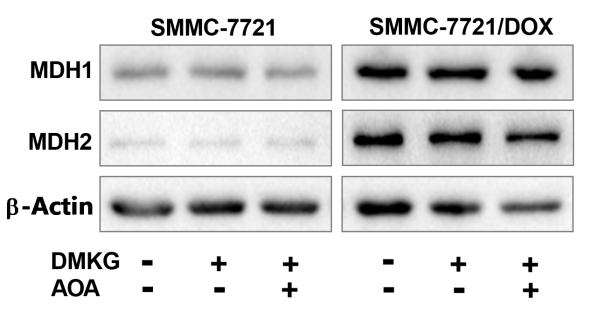


A

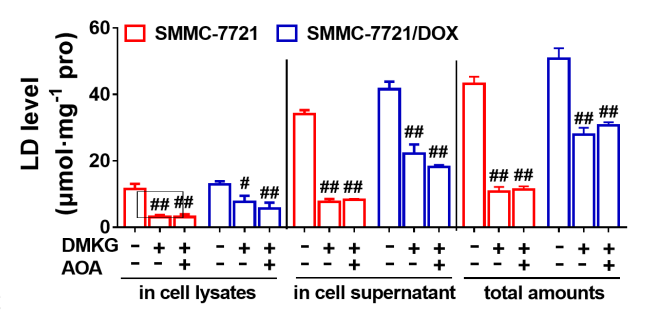

C

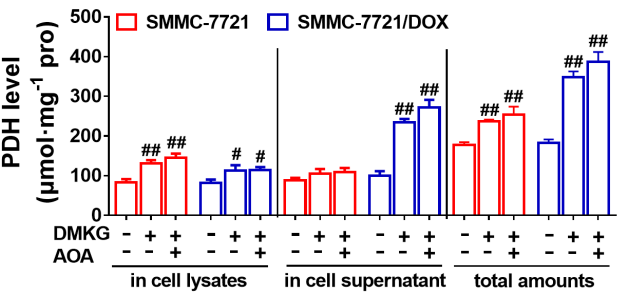

B
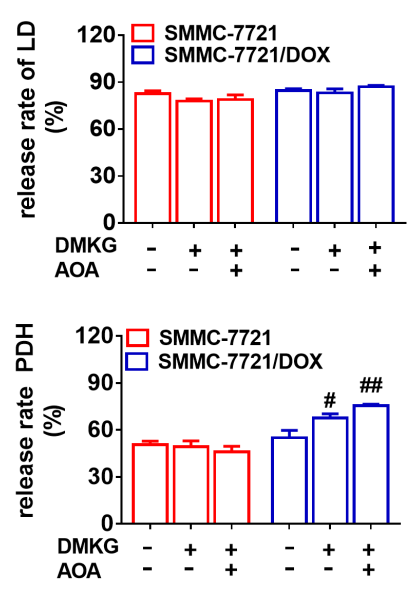

E

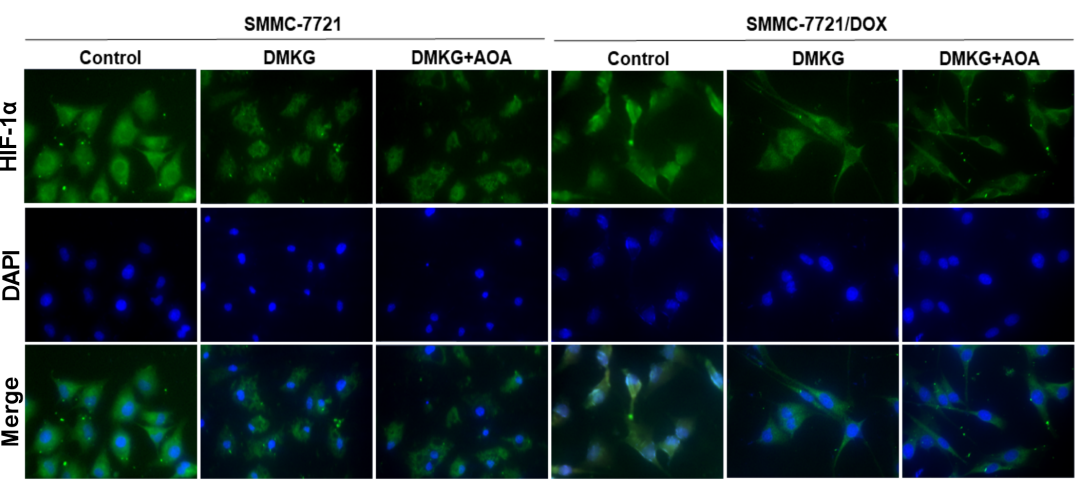

F

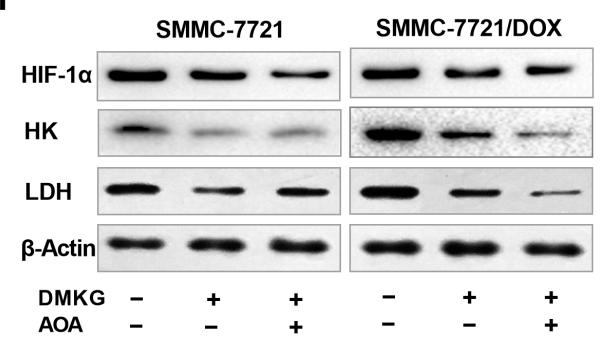

G

H
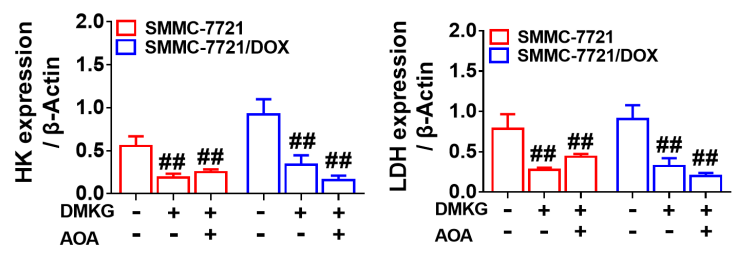

J
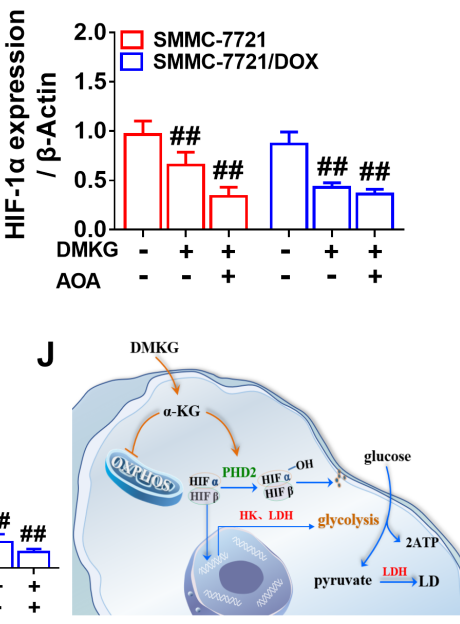
A

SMMC-7721

SMMC-7721/DOX
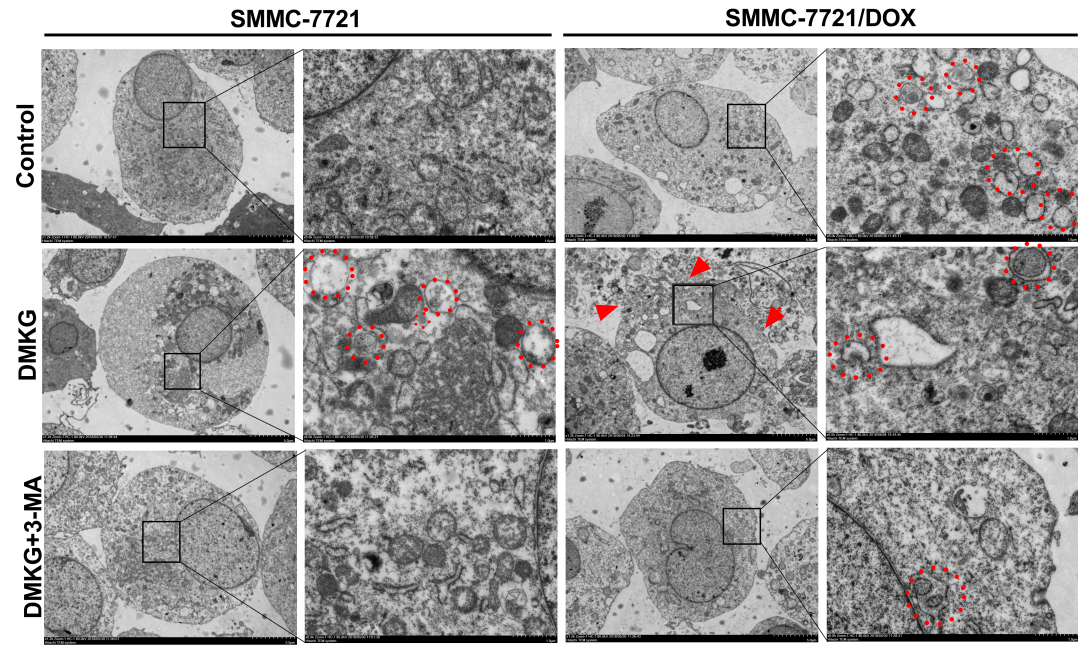

B
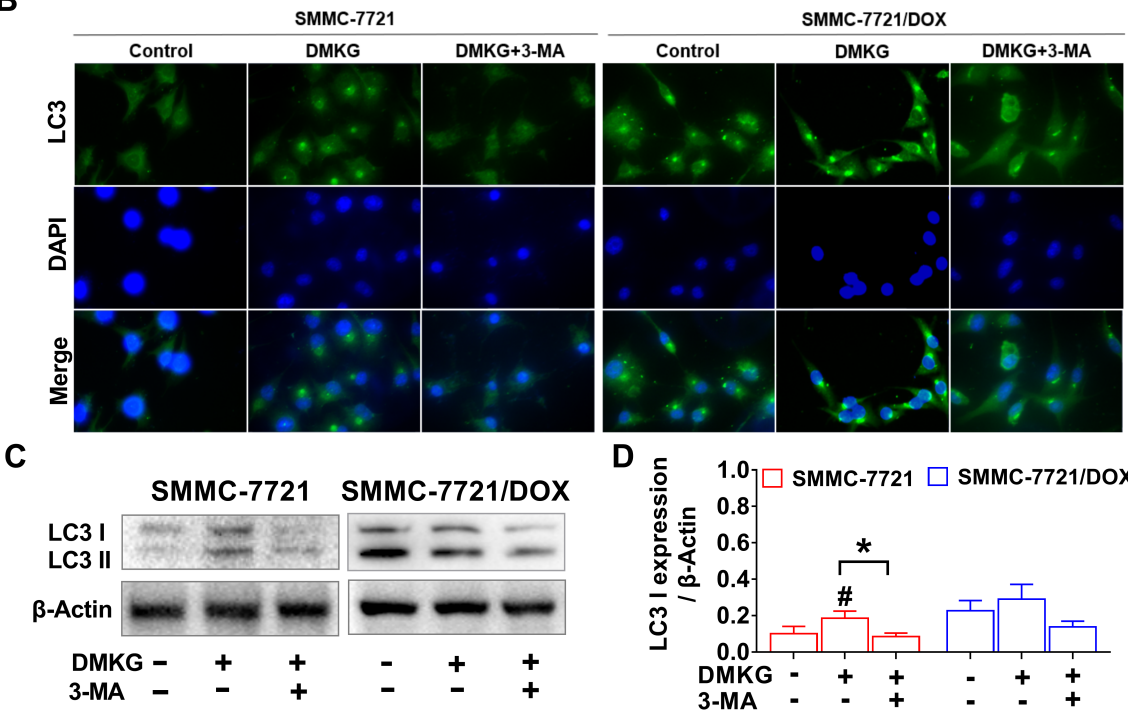

E
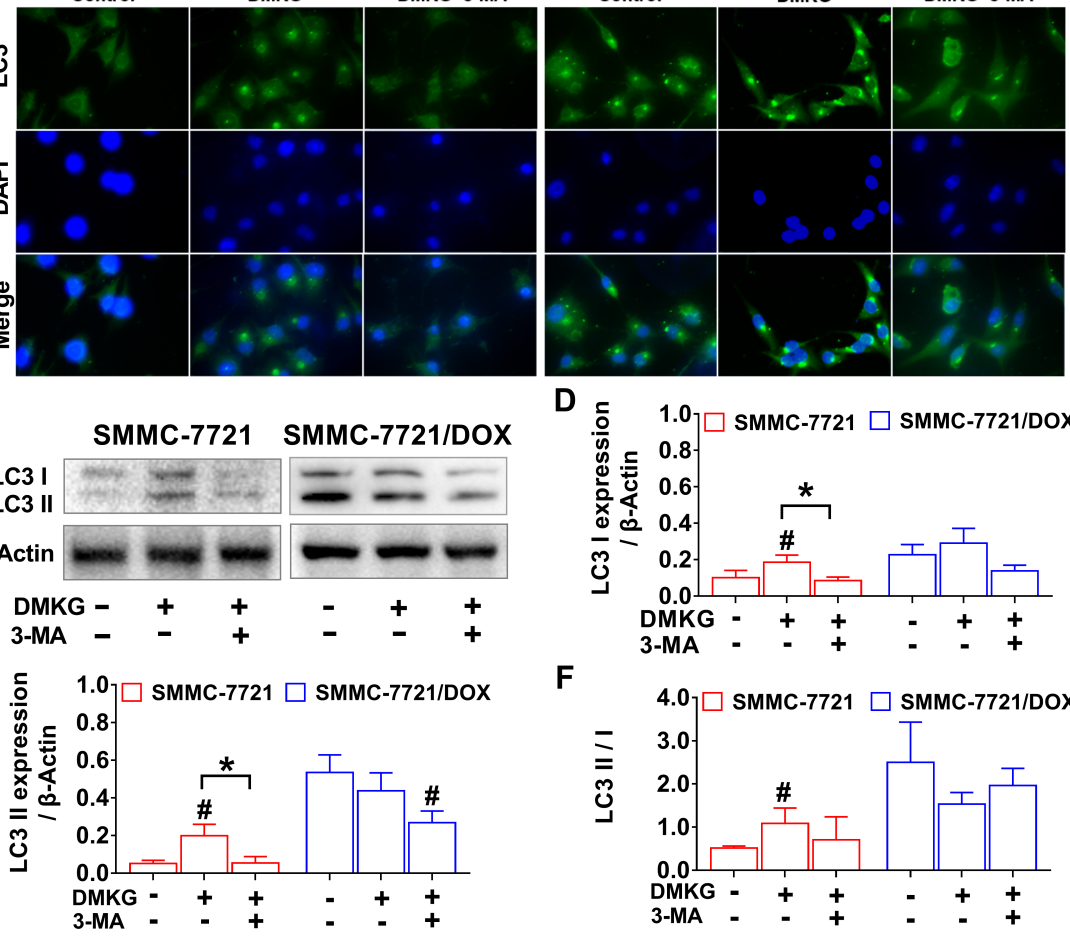

D $\left.\frac{1}{y} \quad 1.0\right] \square$ SMMC-7721 $\square$ SMMC-7721/DOX

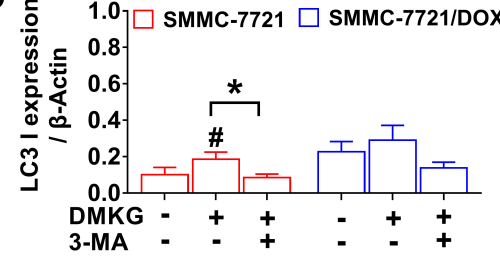

F

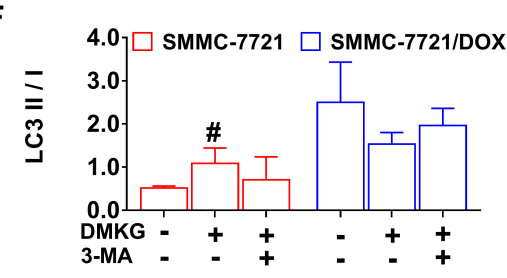



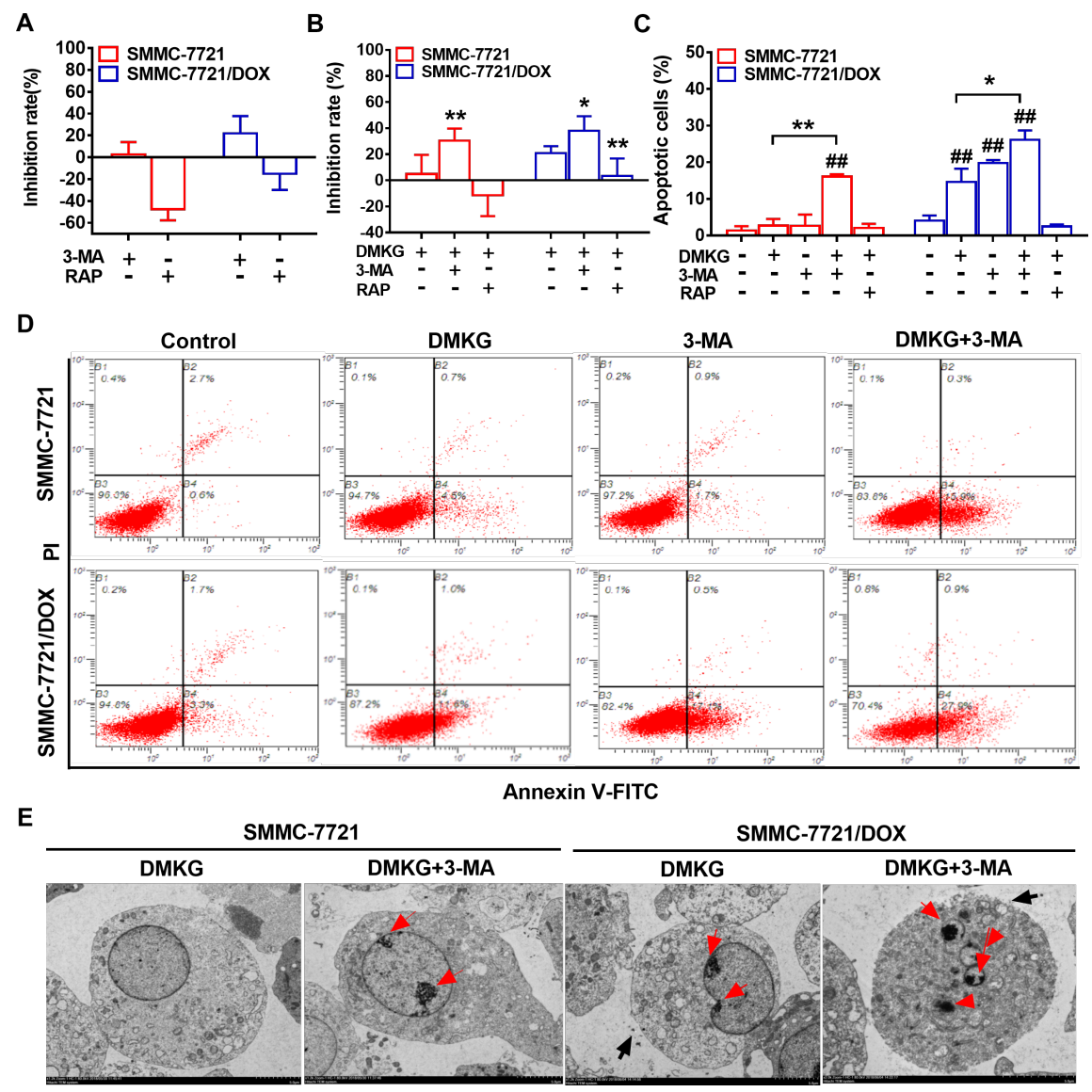
A
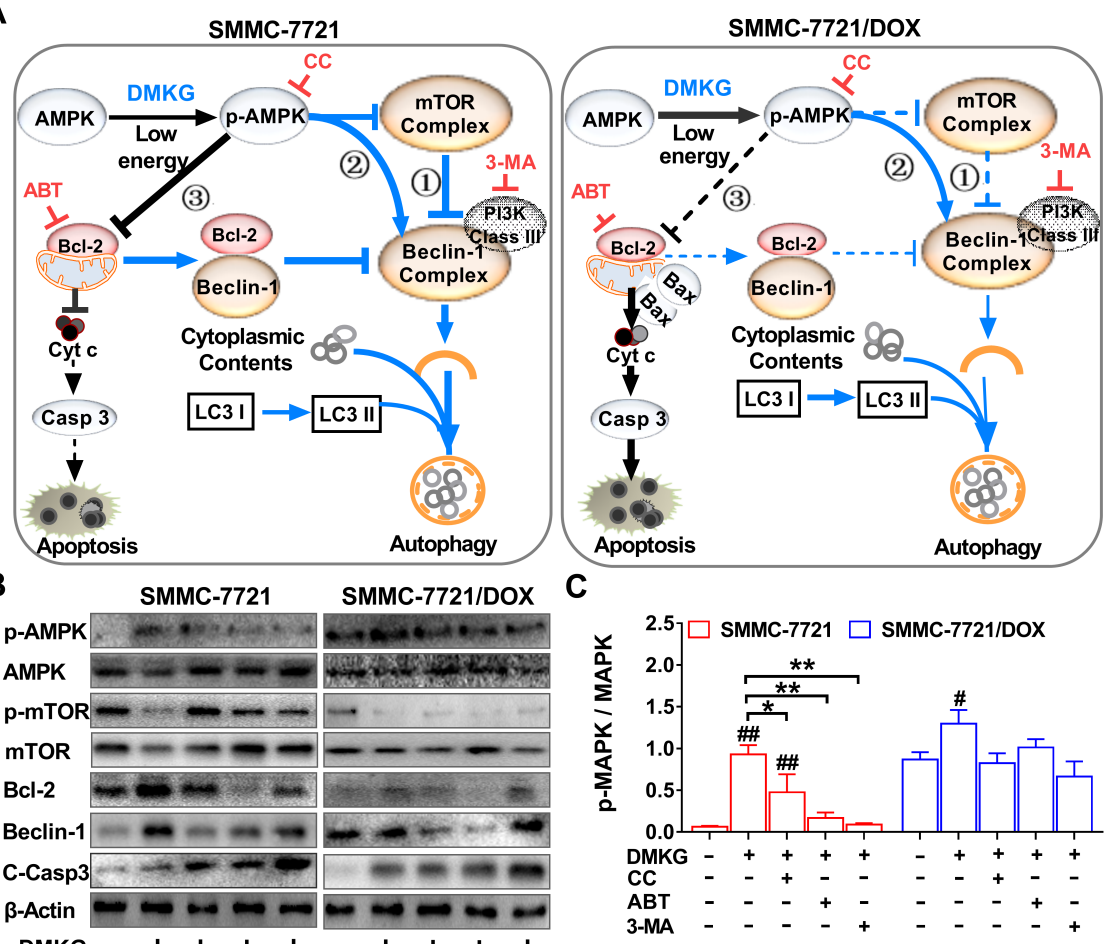

DMKG - + + + + $+++++E$

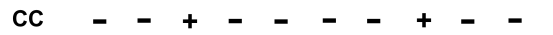
$\begin{array}{lllllllllll}\text { ABT } & - & - & - & + & - & - & - & - & + & - \\ \text { 3-MA } & - & - & - & - & + & - & - & - & - & +\end{array}$

D
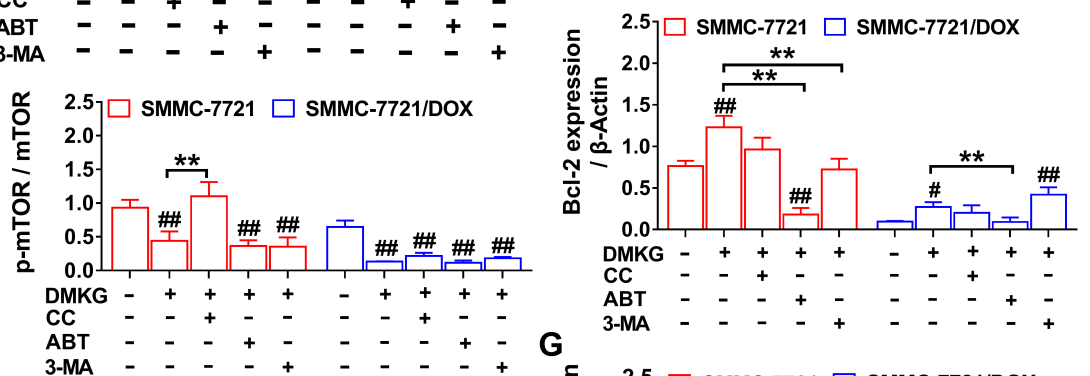

F
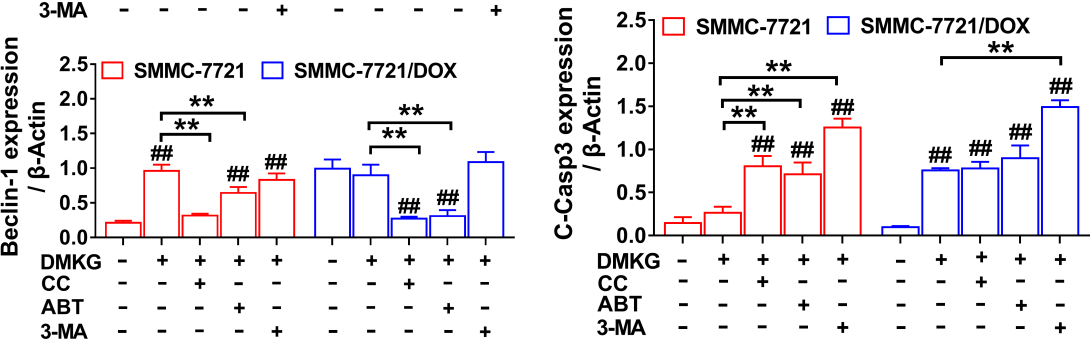